\title{
Drinfeld Doubles for Finite Subgroups of SU(2) and SU(3) Lie Groups
}

\author{
Robert COQUEREAUX ${ }^{\dagger \ddagger}$ and Jean-Bernard ZUBER $\S$ \\ † IMPA \& UMI 2924 CNRS-IMPA, Jardim Botânico, Rio de Janeiro - RJ, 22460-320, Brazil \\ ‡ Aix Marseille Université, CNRS, CPT, UMR 7332, 13288 Marseille, France \\ E-mail: robert.coquereaux@cpt.univ-mrs.fr \\ URL: http://www.cpt.univ-mrs.fr/ coque/ \\ $\S$ LPTHE, CNRS-UMR 7589 and Université Pierre et Marie Curie, \\ 4 place Jussieu, 75252, Paris Cedex 5, France \\ E-mail: zuber@lpthe.jussieu.fr
}

Received December 21, 2012, in final form May 15, 2013; Published online May 22, 2013

http://dx.doi.org/10.3842/SIGMA.2013.039

\begin{abstract}
Drinfeld doubles of finite subgroups of SU(2) and SU(3) are investigated in detail. Their modular data $-S, T$ and fusion matrices - are computed explicitly, and illustrated by means of fusion graphs. This allows us to reexamine certain identities on these tensor product or fusion multiplicities under conjugation of representations that had been discussed in our recent paper [J. Phys. A: Math. Theor. 44 (2011), 295208, 26 pages], proved to hold for simple and affine Lie algebras, and found to be generally wrong for finite groups. It is shown here that these identities fail also in general for Drinfeld doubles, indicating that modularity of the fusion category is not the decisive feature. Along the way, we collect many data on these Drinfeld doubles which are interesting for their own sake and maybe also in a relation with the theory of orbifolds in conformal field theory.
\end{abstract}

Key words: Lie group; fusion categories; conformal field theories; quantum symmetry; Drinfeld doubles

2010 Mathematics Subject Classification: 81R50; 81T40; 20C99; 18D10

\section{Introduction}

Since their introduction by Drinfeld $[15,16]$ quantum doubles of Hopf algebras, in particular of group algebras, have been the subject of some attention, mostly in connection with conformal field theory, representation theory, quantum integrability and topological quantum computation $[9,13,20,31,32,42,48,51]$. Actually the possibility of associating an "exotic Fourier transform" (to become the $S$ matrix of a quantum double) with any finite group was introduced by Lusztig already in [37], see also [36].

The purpose of the present paper is two-fold. As the Drinfeld doubles of specific finite groups may be determined quite explicitly, at least when their order and class number (number of irreps) are not too big, our first purpose is to invite the reader to a tour through a selection of examples taken from the list of finite subgroups of $\mathrm{SU}(2)$ and $\mathrm{SU}(3)$. This is clearly an arbitrary choice, which can be justified from the interest of those subgroups in the construction of the so-called orbifolds models in CFT, and also because their modular data and fusion rules have been determined [6]. Accordingly the reader will find here a selected number of data, tables and graphs (in particular fusion graphs), and we hope that our discussion of the various examples will bring some better understanding in the study of quantum doubles of finite groups. More data are available on request and/or on a dedicated web site ${ }^{1}$.

\footnotetext{
${ }^{1}$ See http://www.cpt.univ-mrs.fr/ coque/quantumdoubles/comments.html.
} 
Secondly, we want to use these data to explore further an issue that has remained somewhat elusive so far: in a recent paper [8], we uncovered identities satisfied by sums of tensor or fusion multiplicities under complex conjugation of representations. This is recalled in detail in Section 2.3 below. These identities were proved for simple Lie algebras of finite or affine type as resulting from a case by case analysis, but no conceptually simple interpretation of that result was proposed. In contrast, these identities were found to fail in a certain number of finite groups. It was suggested [47] that it would be interesting to test these identities on Drinfeld doubles, as they share with affine Lie algebras the property of being modular tensor categories, in contradistinction with finite groups. We shall see below that it turns out that our identities are generally not satisfied by Drinfeld doubles, which indicates that the modular property is not the decisive factor.

The paper is organized as follows. Section 2 recalls a few facts on orbifolds and Drinfeld doubles; it also displays the expressions of modular $S$ and $T$ matrix given in the literature [6,9] and reviews the symmetry properties of $S$, to be used in the following. Section 2.4 presents the sum rules of the fusion coefficients and of the $S$ matrix, to be investigated below. In Section 3, we found it useful to collect a number of "well known" facts on finite groups, that we use in the sequel. Finally in Section 4, we come to the explicit case of finite subgroups of SU(2) and $\mathrm{SU}(3)$. For each of these two Lie groups, a short recapitulation of what is known on their finite subgroups is given, before a case by case analysis of their Drinfeld doubles. The discussion is exhaustive for $\mathrm{SU}(2)$, whereas for $\mathrm{SU}(3)$ we content ourselves with a detailed discussion of the "exceptional" subgroups and a few comments on some subgroups of infinite series. Our results are gathered in Tables 1 and 3. Finally Appendices collect additional comments on finite groups, explicit modular matrices for the particular case of the Drinfeld double of the Klein group $\Sigma_{168}$, and ... a surprise picture.

\section{Orbifolds, doubles and modular data}

\subsection{Remarks about orbifolds models}

In CFT, a general orbifold model is specified by a pair $(\Gamma, k)$ where $\Gamma$ is a Lie group and $k$ is a positive integer, together with a finite subgroup $G$ of $\Gamma$. When $k=0$, orbifold models (called holomorphic) are actually specified by a pair $(G, \omega)$ where $G$ is a finite group and $\omega$ is a cocycle belonging to $H^{3}(G, \mathrm{U}(1))$. The group $G$ can be for instance a subgroup of some given Lie group $\Gamma$ (but the latter plays no role in the construction when $k=0$ ). General orbifold models (with $k \neq 0$ ) are discussed in [31]. These data determine a finite collection of "primary fields", or "simple objects", or irreps, for short. It also determines a fusion ring linearly spanned by the irreps. Those are the so-called chiral data on which one builds a CFT, here an orbifold theory. In BCFT, one has further to specify the kind of "boundary" one considers. A specific boundary type determines a module (also called nimrep) over the fusion ring [1,4].

Using the vocabulary of category theory, one may say that an orbifold model defines a fusion category $\mathcal{A}$ whose simple objects are the primary fields $\sigma_{n}$, labelled by $n$ running from 1 to $r_{A}$. The fusion ring is generated linearly by the primary fields $\sigma_{n}$. The ring structure $\sigma_{m} \sigma_{n}=$ $\sum_{p} N_{m n}^{p} \sigma_{p}$ is specified by non-negative integers $N_{m n}^{p}$. The category is modular: we have $S$ and $T$ matrices, of dimensions $r_{A} \times r_{A}$, representing the generators of the group $\operatorname{SL}(2, \mathbb{Z})$; they obey $S^{2}=(S T)^{3}=C, C^{2}=\mathbb{1}$. The matrix $C$ is called the conjugation matrix. $S$ and $T$ are unitary, $S$ is symmetric. The fusion coefficients $N_{m n}$ are given by the Verlinde formula [50]

$$
N_{m n}^{p}=\sum_{\ell} \frac{S_{m \ell} S_{n \ell} S_{p \ell}^{*}}{S_{1 \ell}} .
$$

In the case $k=0$ (level 0 ) the Lie group $\Gamma$ plays no role, and we set $\mathcal{A}=\mathcal{A}(G, \omega)$. 
A BCFT defines (or is defined by) a module-category $\mathcal{E}$ over $\mathcal{A}$. Its simple objects (called boundary states, in BCFT), labelled by $a$ from 1 to $r_{E}$ are denoted $\tau_{a}$. They generate an abelian group which is a module over the ring of $\mathcal{A}$. Explicitly, $\sigma_{m} \tau_{a}=\sum_{b} F_{m, a} \tau_{b}$. The constants $F_{m, a}^{b}$ are also non-negative integers (whence the acronym nimreps). A BCFT (a choice of $\mathcal{E}$ ) is associated with a symmetric matrix $Z$, of dimensions $r_{A} \times r_{A}$, also with non-negative integer coefficients, that commutes with $S$ and $T$. For this reason $Z$ is called the modular invariant matrix. It is normalized by the condition $Z_{1,1}=1$. For the particular choice $\mathcal{E}=\mathcal{A}$, the boundary states coincide with the primary fields, $r_{E}=r_{A}$, and the modular invariant matrix $Z=\mathbb{1}$ is the unit matrix. The construction of $Z$ from the BCFT/module category $\mathcal{E}$ or vice versa remains in practice a matter of art ....

In general, from any finite group $G$, one can build $\mathcal{A}(G)=\mathcal{A}(G, 0)$ by the so-called Drinfeld double construction $[15,16]$ : it is the representation category of a Hopf algebra $D(G)$ called the Drinfeld double of $G$, or the (untwisted) quantum double of $G$. More generally, from any finite group $G$, together with a cocycle ${ }^{2} \omega$, one can build a fusion category $\mathcal{A}(G, \omega)$ by a method called the twisted Drinfeld double construction. The genuine Hopf algebra $D(G)$ is replaced by a quasi-Hopf algebra $D_{\omega}(G)$. The latter is a quasi-bialgebra, not a bialgebra, because the coproduct is not co-associative.

Remark 1. One may often use various methods to build the same category $\mathcal{A}$, up to equivalence. The Hopf algebra $D(G)$ and the twisted Hopf algebras $D_{\omega}(G)$ have been used in [13] to build $\mathcal{A}(G)$ and $\mathcal{A}(G, \omega)$, but other constructions should be possible.

According to [42] the indecomposable ${ }^{3}$ nimreps $\mathcal{E}$ of $\mathcal{A}(G, \omega)$ or in other words the indecomposable module-categories $\mathcal{E}$ over $\mathcal{A}(G, \omega)$ are parametrized by the conjugacy classes of pairs $(K, \psi)$ where $K \subset G \times G$ is a subgroup, $\psi$ a cohomology class in $H^{2}\left(K, \mathbb{C}^{\times}\right)$, and $K$ is such that the natural extension ${ }^{4} \tilde{\omega}$ of the cohomology class $\omega$ to $H^{3}\left(G \times G, \mathbb{C}^{\times}\right)$is trivial on $K$. Such subgroups $K$ of $G \times G$ are called admissible for $(G, \omega)$. This latter freedom, that usually (not always) changes the modular invariant partition function but not the modular data, was called "discrete torsion" in [49], and in [9]. It is clear that any subgroup $K$ of $G \times G$ is admissible for $\omega=0$.

In what follows we shall only consider holomorphic orbifolds, and moreover often assume that the cocycle $\omega$ is trivial (in other words we shall consider "untwisted holomorphic orbifolds"). For this reason, we shall write "Drinfeld double" instead of "quantum double" in the following. Moreover, we shall not discuss boundary states, BCFT, nimreps, and the like .... Nevertheless, we believe that it was not useless to remind the reader of the above facts concerning modulecategories associated with orbifold models, in order to better understand "where we stand"!

So, what matters for us in this paper is mostly the (modular) fusion category $\mathcal{A}(G)$ associated with the choice of a finite group $G$. It will actually be enough to know how matrices $S$ and $T$ are constructed from some finite group data (see formulae (2) below). The multiplicative structure (the fusion ring coefficients $N_{m n}^{p}$ ) can be obtained from $S$ via Verlinde equations [50]. In particular the Drinfeld double construction (twisted or not), which may be used to obtain the general formulae in Section 2.2 will not be explicitly used in the sequel. In what follows we shall only consider fusion rings obtained from Drinfeld doubles of finite groups, and we therefore drop the general notation $\mathcal{A}(G)$ and write $D(G)$ instead.

\subsection{General properties of Drinfeld doubles}

- We shall call "rank" $r$ the total number of irreps of $D(G)$. As the irreps of $D(G)$ are labelled by pairs $\left([c], \sigma_{c}\right)$, where $[c]$ is a conjugacy class of the group $G$ and $\sigma_{c}$ an irrep of

\footnotetext{
${ }^{2} \operatorname{In} H_{3}(G, \mathbb{Z}) \cong H^{3}\left(G, \mathbb{C}^{\times}\right) \cong H^{3}(G, \mathrm{U}(1))$.

${ }^{3}$ Not equivalent to a direct sum.

${ }^{4} \tilde{\omega}=p_{1}^{\star} \omega-p_{2}^{\star} \omega$, where $p_{i}$ are projections $G \times G \rightarrow G:\left(g_{1}, g_{2}\right) \mapsto g_{i}$.
} 
the centralizer in $G$ of (any representative $c$ of) $[c],,^{5}$ we group together in "blocks" those associated with the same conjugacy class and centralizer. For each example we shall list the number $N_{c}$ of elements (i.e. of irreps $\sigma_{c}$ ) in each block $c$. Their sum is thus equal to the rank $r$. We call "classical" those irreps of the Drinfeld double that correspond to the first block called "classical block", associated with the trivial conjugation class (the centralizer of the identity being $G$ itself, these irreps can be identified with the irreps of the group $G$ ). Their number is equal to the number of conjugacy classes of $G$, that we call the class number.

- Quantum dimensions of irreps, for fusion models built from Lie groups at finite levels (WZW theories), are usually not integers, but quantum dimensions of irreps of doubles of finite groups are always integers. When those irreps are classical, their quantum dimensions coincide with the dimensions of the corresponding irreps of the group.

- If $\chi=\left(c, \sigma_{c}\right)$ is an irrep of $D(G)$, its quantum dimension is $\mu(\chi)$, and the global dimension of $D(G)$ is defined as $|D(G)|=\sum \mu(\chi)^{2}$. In the case of Drinfeld doubles, where the cocycle is trivial, we have $|D(G)|=|G|^{2}$, where $|G|$ is the order of $G$.

- For each of the examples that we consider later, we shall also give the integer $d_{\mathcal{B}}=$ $\sum_{m}\left(\sum_{n, p} N_{m n}^{p}\right)^{2}$ whose interpretation as the dimension of a weak Hopf algebra $\mathcal{B}$ (or double triangle algebra [40]) will not be discussed in this paper, see also [7, 27, 43, 45].

- In writing the $S, T$ and fusion matrices, we sort the irreps as follows. First of all we sort the conjugacy classes according to the increasing order $p$ (in the sense $g^{p}=1$ ) of its representatives. For instance the conjugacy class of the identity (for which $p=1$ ) always appears first. Whenever two classes have the same $p$, their relative order is arbitrarily chosen. Finally, for a given conjugacy class, the irreps of the associated centralizer are ordered (not totally) according to their increasing classical dimension.

- Formulae for $S$ and $T$ :

We copy from [9] the following expressions for the untwisted case. More general expressions that are valid for any twist can be found in the same reference, where they are used to explicitly determine the corresponding $S$ and $T$ matrices in several cases, in particular for the odd dihedral groups (any twist). As recalled above, there is a one to one correspondence between irreps of $D(G)$ and pairs $\left([c], \sigma_{c}\right)$, where $[c]$ is a conjugacy class of $G$, and $\sigma_{c}$ denotes an irrep of the centralizer $C_{G}(c)$ of (any representative $c$ of) class $[c]$ in $G$. Then

$$
\begin{aligned}
S_{\left([c], \sigma_{c}\right)\left([d], \sigma_{d}\right)} & =\frac{1}{\left|C_{G}(c)\right|\left|C_{G}(d)\right|} \sum_{\substack{g \in G \\
c g d g^{-1}=g d g^{-1} c}} \chi_{\sigma_{c}}\left(g d g^{-1}\right)^{*} \chi_{\sigma_{d}}\left(g^{-1} c g\right)^{*} \\
& =\frac{1}{|G|} \sum_{g \in[c], h \in[d] \cap C_{G}(c)} \chi_{\sigma_{c}}\left(x h x^{-1}\right)^{*} \chi_{\sigma_{d}}\left(y g y^{-1}\right)^{*} \\
T_{\left([c], \sigma_{c}\right)\left([d], \sigma_{d}\right)} & =\delta_{c d} \delta_{\sigma_{c} \sigma_{d}} \frac{\chi_{\sigma_{c}}(c)}{\chi_{\sigma_{c}}(e)}
\end{aligned}
$$

where $x$ and $y$ are arbitrary solutions of $g=x^{-1} c x$ and $h=y^{-1} d y$.

In practice, it is more convenient to use a variant of (2) [6]. Let $\mathcal{T}_{c}=\left\{c_{i}\right\}$ (resp. $\left.\mathcal{T}_{d}=\left\{d_{j}\right\}\right)$ be a system of coset representatives for the left classes of $G / C_{G}(c)$ (resp. a system of coset

\footnotetext{
${ }^{5}$ Two elements of the same conjugacy class have isomorphic centralizers.
} 
representatives for the left classes of $\left.G / C_{G}(d)\right)$, then

$$
S_{\left([c], \sigma_{c}\right)\left([d], \sigma_{d}\right)}=\frac{1}{|G|} \sum_{\substack{c_{i}, d_{j} \\ g_{i j}=c_{i} d_{j}^{-1}}}^{\prime} \chi_{\sigma_{c}}\left(g_{i j} d g_{i j}^{-1}\right)^{*} \chi_{\sigma_{d}}\left(g_{i j}^{-1} c g_{i j}\right)^{*},
$$

where the primed sum runs over pairs of $c_{i} \in \mathcal{T}_{c}, d_{j} \in \mathcal{T}_{d}$ that obey $\left[b_{j}^{-1} b b_{j}, a_{i}^{-1} a a_{i}\right]=1$; here $[$,$] is the commutator defined as [a, b]=a^{-1} b^{-1} a b$. This reformulation of (2), also used implicitly in [48], is handy because sets of coset representatives are provided by GAP [23].

\subsection{Symmetries of the $S$ matrix}

- The most conspicuous property of the $S$-matrix in (2) or (3) is its symmetry:

$$
S_{\left([c], \sigma_{c}\right)\left([d], \sigma_{d}\right)}=S_{\left([d], \sigma_{d}\right)\left([c], \sigma_{c}\right)} .
$$

Compare with the case of an ordinary finite group $G$, for which the tensor product multiplicities are given by

$$
N_{r s}^{t}=\sum_{c} \frac{\hat{\chi}_{c}^{r} \hat{\chi}_{c}^{s} \hat{\chi}_{c}^{t *}}{\hat{\chi}_{c}{ }^{1}}
$$

where $\hat{\chi}_{c}^{r}=\sqrt{\frac{|c|}{|G|}} \chi_{c}^{r}$ is the normalized character of irrep $r$ in class $c$, an expression which looks like Verlinde formula (1). In that case, however, there is no reason that the diagonalizing matrix $\hat{\chi}$ of multiplicities be symmetric, and it is generically not. In contrast in a Drinfeld double, that matrix, called now $S$, is symmetric. In other words, there is not only an equal number of classes and irreps in a double, there is also a canonical bijection between them. The origin of that symmetry may be found in a CFT interpretation [13], or alternatively, may be derived directly [32].

- The $S$-matrix has other properties that are basic for our purpose:

- it is unitary, $S . S^{\dagger}=I$;

- its square $S^{2}=C$ satisfies $C . C=I$, i.e. $S^{4}=I$. As recalled above, this is, with $(S . T)^{3}=C$, one of the basic relations satisfied by generators of the modular group. Since $S^{*}=S^{\dagger}=S^{3}=S . C=C . S$, the matrix $C$ is the conjugation matrix, $C_{i j}=\delta_{i \bar{\jmath}}$.

- As just mentioned, under complex conjugation, $S$ transforms as

$$
S_{i j}^{*}=S_{\bar{\imath} j}=S_{i \bar{\jmath}}
$$

where $\bar{\imath}$ refers to the complex conjugate irrep of $i$; in the case of the double, where $i$ stands for $\left([c], \sigma_{c}\right), \bar{\imath}$ stands for $\overline{\left([c], \sigma_{c}\right)}=\left(\left[c^{-1}\right], \overline{\sigma_{c}}\right)$. This follows from the formulae in (2) and [9]. By Verlinde formula this implies that

$$
N_{\bar{\imath}}=N_{i}^{T} .
$$

Thus, for tensor product (fusion), complex conjugation amounts to transposition, a property also enjoyed by (4). Moreover, $N_{\bar{\imath}}=C . N_{i} . C$.

- Other symmetries of the $S$ matrix of the double are associated with the existence of units in the fusion ring. An invertible element in a ring is usually called a unit. A fusion ring is a $\mathbb{Z}_{+}$ring, i.e., it comes with a $\mathbb{Z}_{+}$basis (the irreps), and in such a context, one calls units 
those irreps that are invertible (in the context of CFT, units are generally called simple currents). Therefore if $u$ is a unit, hence an irrep such that $N_{u}$ is invertible, necessarily $N_{u}^{-1}=N_{\bar{u}}=N_{u}^{T}, N_{u}$ is an orthogonal matrix and $\operatorname{det} N_{u}= \pm 1$. In view of (5), $N_{u}$ is an orthogonal integer-valued matrix, hence a permutation matrix, $\left(N_{u}\right)_{i}{ }^{j}=\delta_{J_{u}(i), j}$, where $J_{u}$ is a permutation.

- In the following we denote

$$
\phi_{i}(\ell)=\frac{S_{i \ell}}{S_{1 \ell}}
$$

the eigenvalues of the $N_{i}$ fusion matrix. Note that for a unit $u, \phi_{u}(\ell)$ is a root of unity. Moreover, as $\phi_{u}(1)$ is also a quantum dimension, hence a positive number, this must necessarily be equal to 1 .

- The existence of units entails the existence of symmetries of the fusion graphs, also called representation graphs in the literature, namely the graphs ${ }^{6}$ whose adjacency matrices are the fusion matrices. Each permutation $J_{u}$, for $u$ a unit, acts on irreps in such a way that

$$
\forall i \quad N_{i}=N_{u} N_{\bar{u}} N_{i}=N_{u} N_{i} N_{\bar{u}} \Rightarrow N_{i j}^{k}=N_{i J_{u}(j)}^{J_{u}(k)}
$$

hence may be regarded as an automorphism of the fusion rules and a symmetry of the fusion graphs: on the fusion graph of any $N_{i}$, there is an edge from $j$ to $k$ iff there is one from $J_{u}(j)$ to $J_{u}(k)$. A particular case of such automorphisms is provided by the automorphisms of weight systems in affine algebras used in [8].

- As all irreps of the double, units are labelled by pairs $([c], \psi)$, but here the class $[c]$ is the center $Z(G)$ of $G$, its centralizer is $G$ itself, and $\psi$ is a 1-dimensional irrep of $G$. Indeed, for Drinfeld doubles, the quantum dimension $S_{\left([c], \sigma_{c}\right)([e], 1)} / S_{([e], 1)([e], 1)}$ of an irrep $j=\left([c], \sigma_{c}\right)$ is equal to $|[c]| \times \operatorname{dim}\left(\sigma_{c}\right)$, but for a unit $\left([c], \psi=\sigma_{c}\right)$, the quantum dimension is equal to 1 (see above after (6)), and $c$ is central $(|[c]|=1$ ), so $\psi$ is of degree 1 . The set of the latter is given by the 'abelianization' $G / G^{\prime}$ of $G$, with $G^{\prime}$ the commutator subgroup of $G$. Thus the group of units is isomorphic to $Z(G) \times G / G^{\prime}$.

\subsection{Sum rules for the $S$ matrix}

Let $N_{i j}^{k}$ stand for the multiplicity of irrep $k$ in $i \otimes j$ and let $\bar{\imath}$ refer to the complex conjugate irrep of $i$. According to [8], both in the case of semi-simple Lie groups and in the case of fusion categories defined by a pair $(\Gamma, k)$ (WZW models), we have

$$
\forall i, j \quad \sum_{k} N_{i j}^{k}=\sum_{k} N_{\bar{\imath} j}^{k}
$$

or equivalently

$$
\forall j, k \quad \sum_{i} N_{i j}^{k}=\sum_{i} N_{i j}^{\bar{k}}
$$

In the case of WZW models, where the category is modular, we have shown the above property to be equivalent to the following: if an irrep $j$ is of complex type, then

$$
\Sigma_{j}:=\sum_{i} S_{i j}=0
$$

\footnotetext{
${ }^{6}$ All the graphs given in this paper, as well as many calculations involving fusion matrices, have been obtained with the symbolic package Mathematica [38], interfaced with GAP [23].
} 
and we shall say below that the irrep $j$ has a vanishing $\Sigma$. Actually we have shown in [8] that the last property also holds when $j$ is of quaternionic type.

Defining the charge conjugation matrix $C=S^{2}$ and the path matrix $X=\sum_{i} N_{i}$, it is a standard fact that $C . X . C=X$. Property $\left(7^{\prime}\right)$ reads instead

$$
X=X . C=C . X
$$

The first natural question is to ask whether property (9) holds for finite groups. As noticed in [8], the answer is in general negative (although it holds in many cases). To probe equation (7), we have to look at groups possessing complex representations. In the case of $\mathrm{SU}(2)$ subgroups, equation (7) holds, and this was easy to check since only the cyclic and binary tetrahedral subgroups have complex representations. It was then natural to look at subgroups of SU(3) and we found that (7) holds true for most subgroups of SU(3) but fails for some subgroups like $F=\Sigma_{72 \times 3}$ or $L=\Sigma_{360 \times 3}$. The second property (8) does not make sense for a finite group since there is no invertible $S$ matrix, and Verlinde formula cannot be used.

The next natural question ${ }^{7}$ is to ask if the above properties (7), (8) hold for Drinfeld doubles of finite groups. As we shall see in a forthcoming section, the answer is again negative.

Let us now prove now the following

Proposition 1. For a Drinfeld double: (equation $(7)) \Leftrightarrow \forall j \neq \bar{j}, \Sigma_{j}=0$.

Our proof follows closely the steps of the proof of a similar statement in [8] in the case of finite dimensional or affine Lie algebras, although here neither property is necessarily valid.

- $(8) \Rightarrow(7)$. Suppose that only self-conjugate irreps have a non vanishing $\Sigma$ and use (1) to write

$$
\sum_{k} N_{i j}^{k}=\sum_{\ell} \frac{S_{i \ell} S_{j \ell} \sum_{k} S_{k \ell}^{*}}{S_{1 \ell}}=\sum_{\ell=\bar{\ell}} \frac{S_{i \ell} S_{j \ell} \sum_{k} S_{k \ell}^{*}}{S_{1 \ell}}=\sum_{k} \sum_{\ell=\bar{\ell}} \frac{S_{\bar{\imath} \ell} S_{j \ell} S_{k \ell}^{*}}{S_{1 \ell}}=\sum_{k} N_{\bar{\imath} j}^{k} .
$$

- $(7) \Rightarrow(8)$. Suppose that $\sum_{i} N_{i j}^{k}=\sum_{i} N_{i j}^{\bar{k}}$ for all $i, j, k$. Use again (1) and (7) to write

$$
\begin{aligned}
\left(\sum_{i} S_{i \ell}\right) S_{j \ell} & =\sum_{k} \sum_{i} N_{i j}^{k} S_{k \ell} S_{1 \ell}=\sum_{k} \sum_{i} N_{i j}^{\bar{k}} S_{k \ell} S_{1 \ell} \\
& =\sum_{k} \sum_{i} N_{i j}^{k} S_{\bar{k} \ell} S_{1 \ell}=\sum_{k} \sum_{i} N_{i j}^{k} S_{k \bar{\ell}} S_{1 \bar{\ell}}=\sum_{i} S_{i \bar{\ell}} S_{j \bar{\ell}}=\left(\sum_{i} S_{i \ell}\right) S_{j \bar{\ell}},
\end{aligned}
$$

from which we conclude that if $\sum_{i} S_{i \ell} \neq 0$, then $S_{j \ell}=S_{j \bar{\ell}}$, which cannot hold for all $j$ unless $\ell=\bar{\ell}$ (remember that $S_{j \ell} / S_{1 j}$ and $S_{j \bar{\ell}} / S_{1 j}$ are the eigenvalues of two fusion matrices $N_{\ell}$ and $N_{\bar{\ell}}$ which are different if $\ell \neq \bar{\ell}$ ). Thus, assuming (7) (which is not always granted), if $\ell \neq \bar{\ell}, \sum_{i} S_{i \ell}=0$.

Proposition 2. In any modular tensor category, the complex conjugation is such that properties (7) and (8) are simultaneously true or wrong.

Remark 2. As proved in [8], property (7) hold in the case of Lie groups, and for affine Lie algebras at level $k$ (WZW models), both properties (7) and (8) hold. In the case of Drinfeld doubles of finite groups, it is not always so.

\footnotetext{
${ }^{7}$ We thank Ch. Schweigert for raising that issue.
} 
Whenever the fusion/tensor ring has units, we may state the following

Proposition 3. Consider an irrep $j$ such that there exists a unit $u$ with

$$
\phi_{u}(j)=\frac{S_{u j}}{S_{1 j}} \neq 1
$$

Then (8) holds true: $\sum_{i} S_{i j}=0$.

We write simply, using the fact that $N_{u}=J_{u}$ is a permutation

$$
\sum_{i} S_{i j}=\sum_{i} S_{J_{u}(i) j}=\phi_{u}(j) \sum_{i} S_{i j}
$$

and $\phi_{u}(j) \neq 1 \Rightarrow \sum_{i} S_{i j}=0$.

One finds, however, cases of complex irreps $j$ for which all units $u$ give $\phi_{u}(j)=1$ and Proposition 3 cannot be used. In the examples (see below), we shall encounter the two possibilities:

- Complex irreps (with all $\phi_{u}(j)=1$ ) such that $\Sigma_{j} \neq 0$, hence counter-examples to property (8).

- Vanishing $\Sigma_{j}$ (cf. (8)) for complex, quaternionic and even real irreps $j$ for which all $\phi_{u}(j)=1$. We call such cases "accidental cancellations", by lack of a better understanding.

\section{$3 \quad$ Finite group considerations}

\subsection{About representations, faithfulness, and embeddings}

Before embarking into the study of Drinfeld doubles for finite subgroups of $\mathrm{SU}(2)$ and $\mathrm{SU}(3)$, we need to introduce some terminology and remind the reader of a few properties that belong to the folklore of finite group theory but that we shall need in the sequel.

Any faithful unitary $n$-dimensional (linear) representation of a finite group $G$ on a complex vector space defines an embedding of $G$ into $\mathrm{U}(n)$. An extra condition (the determinant of the representative group elements should be 1 ) is required in order for $G$ to appear as a subgroup of $\mathrm{SU}(n)$. Let us assume, from now on, that $G$ is a subgroup of $\mathrm{SU}(n)$. When the chosen $n$-dimensional representation defining the embedding is irreducible, $G$ itself is called irreducible with respect to $\mathrm{SU}(n)$. When $n>2$, we call embedding representations with respect to $\mathrm{SU}(n)$, those irreps that are $n$-dimensional, irreducible and faithful. The type of a representation can be real, complex, or quaternionic. As the fundamental (and natural) representation of $\mathrm{SU}(2)$ is quaternionic, we adopt in that case a slighly more restrictive definition. For a finite group $G$, isomorphic with an irreducible subgroup of $\mathrm{SU}(2)$, we call embedding representations with respect to $\mathrm{SU}(2)$, those irreps that are 2-dimensional, irreducible, faithful and of quaternionic type. More details can be found in Appendix A.

At times, we shall need the following notion. A finite subgroup $G$ of a Lie group is called Lie primitive (we shall just write "primitive") if it is not included in a proper closed Lie subgroup. Although irreducible with respect to their embedding into $\mathrm{SU}(3)$, some of the subgroups that we shall consider later are primitive, others are not. More details can be found in Appendix B.

The fundamental representation of dimension 3 of $\mathrm{SU}(3)$, or its conjugate, is usually called the natural (or defining) representation, and it is faithful. In this paper we shall mostly, but not always, consider subgroups that are both irreducible and primitive, and the given notion of embedding representation is appropriate (it may be non-unique, see below). However, in some cases, the previous notion of embedding representation should be amended. This is in 
particular so for the cyclic subgroups of SU(2) where no irreducible, faithful, 2-dimensional, and of quaternionic type exists. Notice that for some $\mathrm{SU}(3)$ subgroups, there are cases where the embedding representation - as defined previously - is not of complex type, for the reason that no such representation exists: see below the examples of of $\Delta\left(3 \times 2^{2}\right), \Delta\left(6 \times 2^{2}\right)$, and $\Sigma(60)$, the tetrahedral, octahedral, and icosahedral subgroups of $\mathrm{SO}(3) \subset \mathrm{SU}(3)$, which have no complex 3-dimensional irrep. They are not primitive subgroups of $\mathrm{SU}(3)$.

In the present paper we are not so much interested in fusion graphs associated with finite groups $G$, rather we are interested in their Drinfeld doubles $D(G)$. There is one fusion graph for each irrep of $D(G)$ and it is of course out of question to draw all of them in this paper. For this reason, we are facing the problem of which representation to select. As recalled above, irreps of $D(G)$ are labeled by pairs (a conjugacy class of $G$ and an irrep of the corresponding centralizer). One special conjugacy class is the class of the neutral element $\{e\}$ of $G$, which has only one element, its centralizer being $G$ itself. As we are mostly interested in irreducible embeddings that define finite subgroups $G$ of $\mathrm{SU}(n)$ for $n=2$ or 3 , the selected representation of $D(G)$ will (with a few exceptions, see later) be of the type $(\{e\}, \rho)$ with $\rho$ chosen among the irreducible faithful representations of $G$ of the appropriate dimension $n$. We shall call "embedding irrep" of the Drinfeld double of $G$, any pair $(\{e\}, \rho)$ where $\rho$ is an embedding representation for $G$, with the above meaning.

\subsection{About faithfulness, and connectedness of fusion graphs}

Faithfulness of the selected embedding representation (of $G$ ) can be associated with several concepts and observations related to connectedness properties of the associated fusion graph of $G$ or of $D(G)$ : the former is connected whereas the latter appears to have a number of connected components equal to the class number of $G$.

Fundamental representations of a simple complex Lie group or of a real compact Lie group can be defined as those irreps whose highest weight is a fundamental weight. These irreps generate by fusion (tensor product) all irreps. Still for Lie groups, and more generally, we may ask for which irreducible representation $\rho$, if any, fundamental or not, can we obtain each irreducible representation as a subrepresentation of a tensor power $\rho^{\otimes k}$, for some $k$. This is a classical problem and the answer is that $\rho$ should be faithful [30]; in the same reference it is shown that, except when $G=\operatorname{Spin}(4 n)$, there exist faithful irreducible representations for all the simple compact Lie groups.

In the theory of finite groups, there is no such notion as being fundamental, for an irreducible representation. However, one can still ask the same question as above, and the result turns out to be the same (Burnside, as cited in [11]): if $G$ is a finite group and $\rho$ is a faithful representation of $G$, then every irreducible representation of $G$ is contained in some tensor power of $\rho$. In other words, the fusion graph associated with a faithful representation of a finite group is connected, since taking tensor powers of this representation amounts to following paths on its fusion graph, and all the irreps appear as vertices.

Let $H$ be a subgroup of the finite group $G$ and let $\rho$ be a faithful representation of $G$. Then $\rho_{H}$, the restriction of $\rho$ to $H$, may not be irreducible, even if $\rho$ is, but it is clearly faithful: its kernel, a subgroup of $H$, is of course trivial since the kernel of $\rho$ was already trivial in the first place. Therefore every irreducible representation of $H$ is contained in some tensor power

of $\rho_{H}$. Writing $\rho_{H} a=\sum_{b} F_{\rho, a}^{b} b$, where $a, b, \ldots$ are irreps of $H$, defines a matrix $F_{\rho}$ which is the adjacency matrix of a graph. This (fusion) graph is connected, for the same reason as before. Notice that $\rho_{H}$ itself may not appear among its vertices since it may be non irreducible.

As mentioned previously every representation $\rho$ of $G$ determines a representation $(e, \rho)$ of $D(G)$. The representation rings for the group $G$ and for the algebra $D(G)$ are of course different, the fusion coefficients of the former being obtained from its character table, those of 
the latter from the modular $S$-matrix and the Verlinde formula, but the former can be considered as a subring of the latter. Since irreps of the double fall naturally into blocks indexed by conjugacy classes, we expect that the fusion graph of an embedding irrep of $D(G)$ will have several connected components, one for each conjugacy class, i.e., a number of components equal to the number of classes of $G$, i.e., to the class number. This graph property is actually expected for all the irreps of $D(G)$ stemming from $(z, \rho)$, with $z \in Z(G)$ and a faithful irreducible representation $\rho$ of $G$. Indeed, the usual character table of $G$ can be read from the $S$ matrix in the following way: extract from $S$ the submatrix made of its first $\ell$ rows (the "classical irreps" $r$ ), in the latter keep only the first column of each of the $\ell$ blocks (corresponding to different classes $c$ of $G$ ) and finally multiply these columns by $|G| /|c|$, resp. $\sqrt{|G| /|c|}$; this yields the matrix $\chi_{c}^{r}$, resp. $\hat{\chi}_{c}^{r}$ defined in (4). A similar construction applies to the character tables pertaining to the different centralizers of conjugacy classes, which may also be extracted from the $S$-matrix - the latter is much more than a simple book-keeping device for the character tables of the different centralizers of conjugacy classes since it couples these different blocks in a non-trivial way. On the basis of all examples that we have been considering, and in view of the above discussion, we conjectured

The fusion graph of an embedding irrep of $D(G)$ has $\ell$ connected components, with $\ell$, the class number, equal to the number of irreps or of conjugacy classes of $G$.

A formal proof of this property, that we shall not give ${ }^{8}$, can exploit, in the language of fusion categories, the relation between the representation rings of $G$ and $D(G)$, in particular a generalization (see for instance [51]) of the mechanism of induction and restriction. Notice that an embedding fusion graph, for the group $G$ (the fusion graph of an embedding representation) can be obtained by selecting the connected component of $(e, 1)$ in the graph of the corresponding embedding graphs of its double. Since it describes a faithful representation, the number of vertices of this connected component is also equal to the class number of $G$.

\subsection{Additional remarks}

In general, a finite group $G$ may have more than one irreducible faithful representation of given dimension $n$ (up to conjugation, of course); for instance a representation of complex type will always appear together with its complex conjugate in the table of characters, but there are groups that possess several conjugated pairs of inequivalent faithful irreps of complex type, with the same dimension, and they may also possess self-dual (i.e., real or quaternionic) faithful irreps, also of the same dimension, see the example of $\Sigma_{168} \times \mathbb{Z}_{3}$ below. There are even finite groups that have more than one pair of faithful irreducible representations of complex type, with different dimensions, for instance the SU(3) subgroup $\Sigma_{168} \times \mathbb{Z}_{3}$ possesses faithful irreps of complex type in dimensions $3,6,7,8$. With the exception of cyclic groups, all the finite subgroups of $\mathrm{SU}(2)$ have at least one 2-dimensional irreducible faithful representation of quaternionic type.

The smallest binary dihedral group (or quaternion group) $\hat{D}_{2}$ has only one such irrep, and it is quaternionic, but higher $\hat{D}_{n}$ 's have 2-dimensional irreps that may be real and non faithful or quaternionic and faithful; as it was explained, we shall call embedding representations with respect to $\mathrm{SU}(2)$, only those that are faithful. One can check on Fig. 1 giving for binary dihedral groups all the fusion graphs associated with 2-dimensional representations of the classical block of the Drinfeld double, that, as expected, only the faithful ones have a number of components equal to the number of classical irreps.

The binary tetrahedral group $\hat{T}$ has three 2-dimensional irreps and they are faithful: only one (that we label 4) is quaternionic, whereas those labelled 5 and 6 are complex (and conju-

\footnotetext{
${ }^{8}$ Note added: As noticed by an anonymous referee to whom we are deeply indebted, such a proof actually follows from simple considerations making use of results of [5, 17], in particular of the formula $(e, \rho) \otimes(a, \delta)=$ $\left(a, \rho \downarrow_{C_{G}(a)}^{G} \otimes \delta\right)$ where $\rho$ is an irrep of $G$ and $\delta$ an irrep of the centralizer $C_{G}(a)$ of $a \in G$.
} 

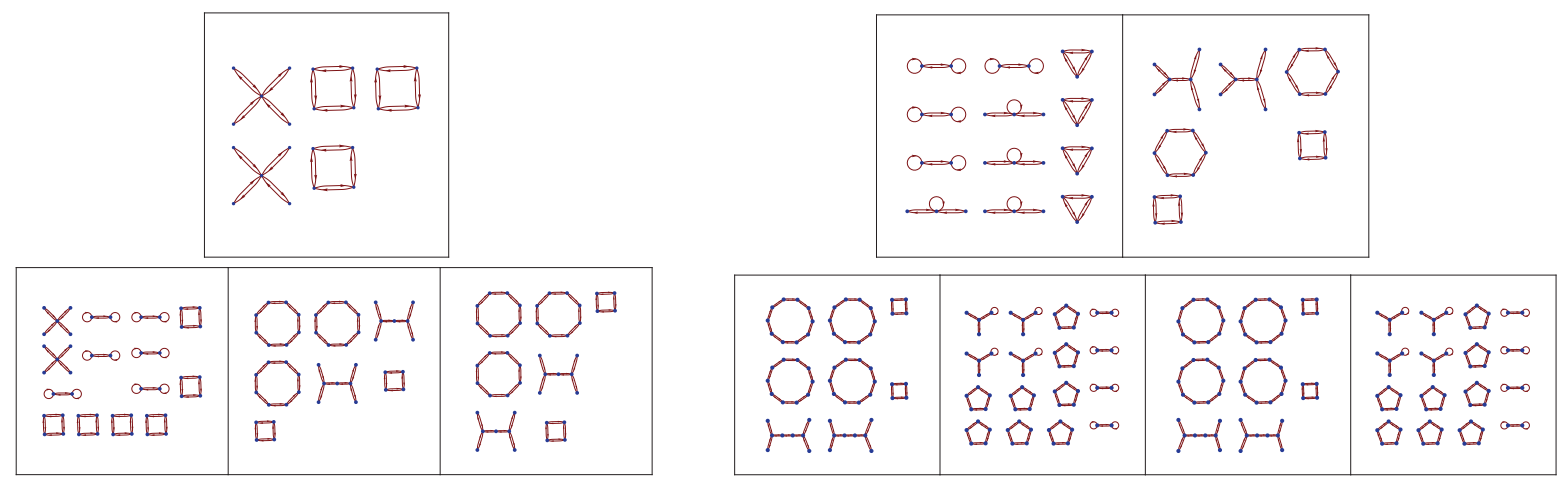

Figure 1. Fusion graphs of the classical irreps of dimension 2 for the binary dihedral $\widehat{D}_{2}, \widehat{D}_{3}, \widehat{D}_{4}, \widehat{D}_{5}$. The graphs of the faithful irreps have a number of connected components equal to the class number (resp. $5,6,7,8)$.

gated). The fusion graph associated with $N_{4}$, that we call embedding representation with respect to $\mathrm{SU}(2)$, is the affine $E_{6}$ graph (by McKay correspondence, see below); the fusion graphs associated with $N_{5}$ or $N_{6}$ are also connected graphs (these representations are faithful!), but they have rather different features. The binary octahedral group has also three 2-dimensional irreps, but one $\left(N_{3}\right)$ is real and not faithful, the other two, $N_{4}$ and $N_{5}$, that we call embedding irreps, are both faithful and quaternionic. The binary icosahedral group has two 2-dimensional irreps $\left(N_{2}, N_{3}\right)$, they are both faithful and quaternionic.

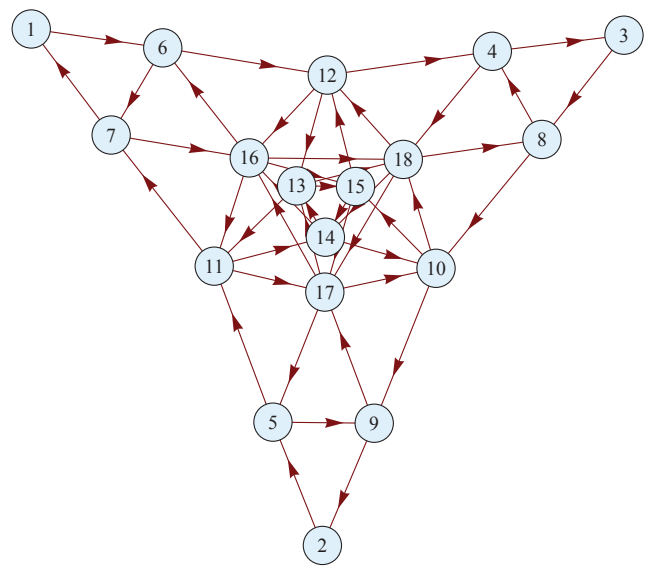

Figure 2. Fusion graph $N_{6}$ of the subgroup $\Sigma_{168} \times \mathbb{Z}_{3}$.

The case of $\mathrm{SU}(3)$ is a bit more subtle because irreducible subgroups like $\Sigma_{60}$ may be associated with imprimitive embedding. At this place we just illustrate on one example the importance of the faithfulness requirement: the group $\Sigma_{168} \times \mathbb{Z}_{3}$ has 18 irreps, six of them, actually three pairs of complex conjugates, labelled 4, 5,6, 7, 8, 9 are of dimension 3 , but the irreps of the pair $(4,5)$ are not faithful whereas the two pairs $(6,7)$ and $(8,9)$ are. As it happens the fusion graphs associated with the faithful representations (namely those labeled $6,7,8,9,11$, $12,14,15,17,18)$ are connected; this is not so for the others, in particular for the 3-dimensional irreps labelled 4 and 5 . So the natural (or embedding) irreps, with respect to $\mathrm{SU}(3)$, are 6,7 , 8,9 and we may choose to draw the fusion graph of $N_{6}$ for instance (see Fig. 2). The Drinfeld double of this group $\Sigma_{168} \times \mathbb{Z}_{3}$ has 288 irreps, its first block has 18 irreps, as expected, that we label as for the finite group itself, and again, we shall give the fusion graph of $N_{6}$. This graph is no longer connected but its number of connected components is equal to the number of blocks 
(also the number of conjugacy classes, or of irreps of $G$ itself, namely 18), see below Fig. 17 . These features are shared by $N_{6}, N_{7}, N_{8}, N_{9}$ but not by $N_{4}$ or $N_{5}$.

The conjugacy class determined by every central element of $G$ contains this element only, and the centralizer of a central element of $G$ is $G$ itself, therefore the irreps of $G$ should appear $|Z(G)|$ times in the list of irreps of $D(G)$, where $Z(G)$ is the centre of $G$. For example, the group $\Sigma_{36 \times 3}$ has four pairs of 3-dimensional complex conjugated irreps (every one of them can be considered as an embedding irrep), and its center is $\mathbb{Z}_{3}$, so we expect that $3 \times(4 \times 2)=24$ irreps, among the 168 irreps of the double, will have similar properties, in particular the same quantum dimensions (namely 3 , since, as we know, quantum dimensions of irreps of Drinfeld doubles are integers), and also isomorphic fusion graphs (i.e., forgetting the labeling of vertices).

\section{Drinfeld doubles (examples)}

In the following, we review a certain number of finite subgroups of $\mathrm{SU}(2)$ and $\mathrm{SU}(3)$, giving for each its essential ${ }^{9}$ data, the order of the group $G$, its name in GAP nomenclature, class number, and for its Drinfeld double $D(G)$, the rank (number of irreps), $N_{c}$ the dimensions of the blocks, and quantum dimensions. In order to shorten the often long lists of quantum dimensions, we write $n_{s}$ when $n$ is repeated $s$ times in a list ${ }^{10}$. We also give the label(s) of the embedding representation(s), the fusion graph of which is then displayed. On the connected component of the identity representation (i.e., the "classical block") one recognizes the corresponding fusion graph of the original group $G$. Moreover one checks that the total number of connected components of any of these embedding fusion graphs equals the class number of $G$, as conjectured in Section 3.1.

\subsection{Drinfeld doubles of finite subgroups of $\mathrm{SU}(2)$}

\subsubsection{Remarks about finite subgroups of SU(2)}

General. $\operatorname{Spin}(3)=\mathrm{SU}(2)$ is the (universal) double cover of $\mathrm{SO}(3)$. $\mathbb{Z}_{2}$ is the center of $\mathrm{SU}(2)$. With every subgroup $\Gamma$ of $\mathrm{SO}(3)$ is associated its binary cover $\widehat{\Gamma}$, a subgroup of $\mathrm{SU}(2)$. Then, of course, $\Gamma \cong \widehat{\Gamma} / \mathbb{Z}_{2}$. Finite subgroups of $\mathrm{SU}(2)$ are of the following kind: cyclic, binary dihedral, binary tetrahedral, binary octahedral, binary icosahedral. The fusion graphs presented below refer to the fusion matrix of the embedding representation, unless stated otherwise.

\section{Cyclic groups.}

- $\mathbb{Z}_{2}$ cannot be a subgroup of $\mathbb{Z}_{q}$ when $q$ is odd (the order of a subgroup should divide the order of the group!) but it is a subgroup of $\mathbb{Z}_{q}$ when $q$ is even $(q=2 p)$, and $\mathbb{Z}_{p}=\mathbb{Z}_{q} / \mathbb{Z}_{2}$.

- Cyclic groups $\mathbb{Z}_{q}$, for all $q \in \mathbb{N}$, are subgroups of $\mathrm{SO}(3)$ and also subgroups of $\mathrm{SU}(2)$.

- When $q$ is even $(q=2 p)$, we may consider the subgroup $\mathbb{Z}_{q}$ of $\mathrm{SU}(2)$ as the binary group corresponding to the subgroup $\mathbb{Z}_{p}$ of $\mathrm{SO}(3)$ (and this $p$ can be even or odd).

- When $q$ is odd, $\mathbb{Z}_{q}$ is a subgroup of $\mathrm{SU}(2)$, but not the binary of a subgroup of $\mathrm{SO}(3)$.

- The homology or cohomology groups $H_{2}\left(\mathbb{Z}_{q}, \mathbb{Z}\right) \cong H^{2}\left(\mathbb{Z}_{q}, \mathbb{C}^{\times}\right) \cong H^{2}\left(\mathbb{Z}_{q}, U(1)\right)$ are trivial ("the Schur multiplier is trivial"). Hence any Schur cover of $\mathbb{Z}_{q}$ is equal to itself. Nevertheless, the cyclic group of order $2 p$ can be considered as an extension, by $\mathbb{Z}_{2}$, of a cyclic group of order $p$. $\mathbb{Z}_{2 p}$ is the binary cover of $\mathbb{Z}_{p}$ but it is not a Schur cover of the latter.

\section{Dihedral groups and their binary covers.}

- Dihedral groups $D_{n}$, of order $2 n$ are, for all $n \in \mathbb{N}$, subgroups of $\operatorname{SO}(3)$.

\footnotetext{
${ }^{9}$ For "famous" groups of relatively small order, one can retrieve a good amount of information from [26].

${ }^{10}$ The subindex $s$ therefore does not refer to an $s$-integer (all our quantum dimensions are integers in this paper!), and $s$ does not denote a multiplicity in the usual sense.
} 
- The smallest one, $D_{1}$, of order 2 , is isomorphic to $\mathbb{Z}_{2}$ and is usually not considered as a dihedral group.

- In the context of the study of the covering $\mathrm{SU}(2) \rightarrow \mathrm{SO}(3)$, all of these groups $D_{n}$ can be covered by subgroups $\widehat{D}_{n}$ of $\mathrm{SU}(2)$ of order $4 n$, called binary dihedral groups (they are also called dicyclic groups).

- The Schur multiplier of dihedral groups $H_{2}\left(D_{n}, \mathbb{Z}\right) \cong H^{2}\left(D_{n}, \mathbb{C}^{\times}\right) \cong H^{2}\left(D_{n}, U(1)\right)$ is trivial when $n$ is odd, and is $\mathbb{Z}_{2}$ when $n$ is even. Nevertheless, in both cases (even or odd) one may consider the corresponding binary dihedral groups (of order $4 n$ ) that are subgroups of $\mathrm{SU}(2)$.

The tetrahedral group $T$ and its binary cover $\widehat{T} \cong \mathrm{SL}(2,3)$. The tetrahedral group is $T=\widehat{T} / \mathbb{Z}_{2} \cong A_{4}$ (alternating group on four elements).

The cubic (or octahedral) group $O$ and its binary cover $\widehat{O}$. The octahedral group is $O=\widehat{O} / \mathbb{Z}_{2} \cong S_{4}$ (symmetric group on four elements) and its Schur multiplier is $\mathbb{Z}_{2}$. Warning: $\widehat{O}$ is not isomorphic to GL $(2,3)$, although this wrong statement can be found in the literature. It can be realized in $\mathrm{GL}(2,9)$, as the matrix subgroup generated by $a=((-1,1),(0,-1))$ and $b=((-u,-u),(-u, 0))$, where $u$, obeying $u^{2}=-1$, is an element added to $F_{3}$ to generate $F_{9}$. We thank [41] for this information. If $w$ generates $F_{9}$ (so $w^{9}=w$ ), we take $u=w^{2}$. To our knowledge, this is the smallest realization of $G$ as a matrix group, and we used it to calculate the Drinfeld double of the binary octahedral group. Using GAP nomenclature, $\widehat{O}$ can be recognized as SmallGroup $(48,28)$.

The icosahedral group $I$ and its binary cover $\widehat{I} \cong \operatorname{SL}(2,5)$. The icosahedral group is $I \cong A_{5}$ (alternating group on five elements), the smallest non-abelian simple group, and its Schur multiplier is $\mathbb{Z}_{2}$.

Remarks about finite subgroups of $\mathrm{SO}(3)$ and $\mathrm{SU}(2)$ (continuation).

- Dihedral groups $D_{n}$, of order $2 n$, with $n$ odd, and cyclic groups (all of them) are the only subgroups of $\mathrm{SO}(3)$ that have trivial Schur multiplier.

- The polyhedral groups are subgroups of SO(3). The binary polyhedral groups are subgroups of SU(2). The so-called "full polyhedral groups" (that we do not use in this paper) are subgroups of $\mathrm{O}(3)$ and should not be confused with the binary polyhedral groups. Notice however that the full tetrahedral group is isomorphic to the octahedral group (both being isomorphic to $S_{4}$ ).

- The Schur multiplier of the exceptional polyhedral groups (tetrahedral, cube and icosahedral) are non trivial (they are equal to $\mathbb{Z}_{2}$ ) and, for them, the binary cover can be used as a Schur cover (however a finite group may have several non isomorphic Schur covers, see the remarks in Appendix C). The same is true, when $n$ is even, for the dihedral groups $D_{n}$.

- All discrete finite subgroups $G$ of $\mathrm{SU}(2)$ have trivial second cohomology $H^{2}(G, \mathbb{C})=1$ and thus trivial Schur multiplier.

\section{About the fusion graphs of the doubles.}

- We shall focus our attention on the embedding irreps (or one of them if there are several) as defined at the beginning of this section and on its fusion matrix and graph. Its label will be called "embedding label" in the Tables below, and its fusion graph called the embedding graph.

- In all these embedding graphs, the connected part relative to the "classical" representations is isomorphic to an affine Dynkin diagram of type $A^{(1)}, D^{(1)}, E_{6}^{(1)}, E_{7}^{(1)}, E_{8}^{(1)}$. This is of course nothing else than a manifestation of the celebrated McKay correspondence in this context [39]. 
- Another comment is that in Drinfeld doubles of binary groups, the classes of the identity $I$ and of its opposite $-I$ have the same centralizer, v.i.z. the group itself. To any embedding representation of the double (in the block of $I$ ) there is an associated irreducible in the block of $-I$. We found it useful to draw the two graphs of these two irreducibles together in different colors in several cases, see below Figs. 6, 8, 10.

\subsubsection{Drinfeld doubles of the (binary) cyclic subgroups}

We consider the example of $\mathbb{Z}_{6}$.

Order of the group: 6

GAP nomenclature: SmallGroup(6,2)

Class number: $\ell=6$

Rank: (defined as the number of irreps of $D(G)$ ) $r=36$

Numbers $N_{c}$ of irreps of $D(G)$ in each block $=6,6,6,6,6,6$

Quantum dimensions: $\left(1_{6} ; 1_{6} ; 1_{6} ; 1_{6} ; 1_{6} ; 1_{6}\right)$ in which we use a shorthand notation: $p_{s}$ indicates that there are $s$ irreps of dimension $p$; different blocks are separated by semi-colons.

$d_{\mathcal{B}}=2^{6} 3^{6}$

Embedding labels: $4 \oplus 6$.

As explained above, in such an abelian group in which irreps are one-dimensional, the 2dimensional embedding representation is the direct sum of two irreps: its is reducible as a complex representation, irreducible as a real one.) See the fusion graph of $N_{4}$ on Fig. 3. The "embedding graph" associated with $N_{4}+N_{6}$ looks the same, but with unoriented edges between vertices.
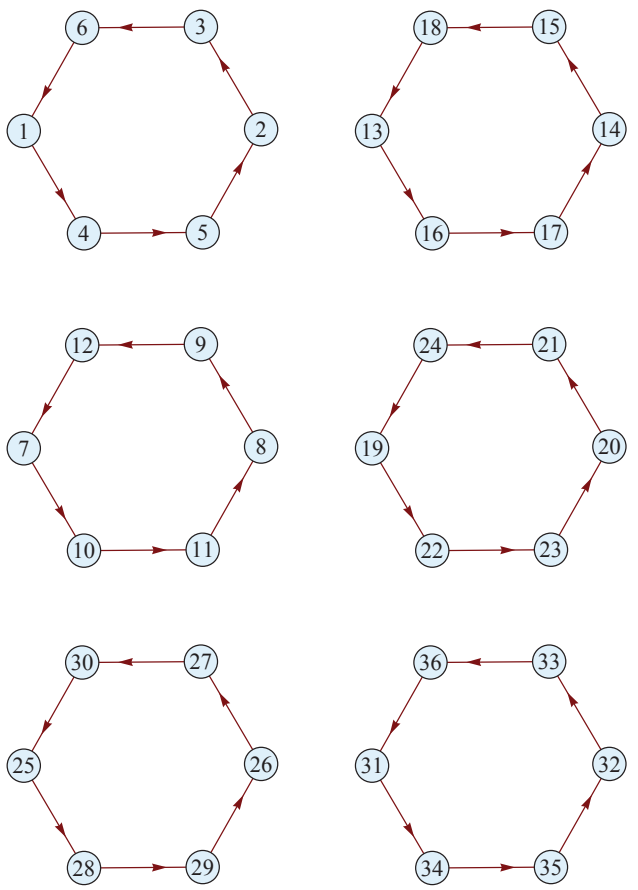

Figure 3. Fusion graph $N_{4}$ of the Drinfeld double of the cyclic group $\mathbb{Z}_{6}$.

Take $G=\mathbb{Z}_{p}$, with $p$ odd. This is not a binary cover, but it is nevertheless a subgroup of $\mathrm{SU}(2)$. Only the trivial representation is of real type. All others are complex. Observation: Only the trivial representation has non-vanishing $\Sigma$. In particular, all complex representations of the double have vanishing $\Sigma$. The sum rule (7) holds.

Take $G=\mathbb{Z}_{p}$, with $p$ even (so $G$ is a binary cover). There are several representations of real type, all the others being complex. Observation: Only the trivial representation has non- 
vanishing $\Sigma$. In particular, all complex representations of the double have vanishing $\Sigma$. The sum rule (7) holds.

\subsubsection{Drinfeld doubles of the binary dihedral subgroups}

We consider the example of $\widehat{D}_{5}$.

Order of the group: 20

GAP nomenclature: SmallGroup $(20,1)$

Class number: $\ell=8$

Rank: $r=64$

$N_{c}=8,8,4,4,10,10,10,10$

Quantum dimensions: $\left(1_{4}, 2_{4} ; 1_{4}, 2_{4} ; 5_{4} ; 5_{4} ; 2_{10} ; 2_{10} ; 2_{10} ; 2_{10}\right)$

$d_{\mathcal{B}}=2^{8} 4363^{1}$

Embedding labels: 5, 7. See the fusion graph of $N_{5}$ on Fig. 4. That of $N_{7}$ looks very similar, up to a permutation of labels.
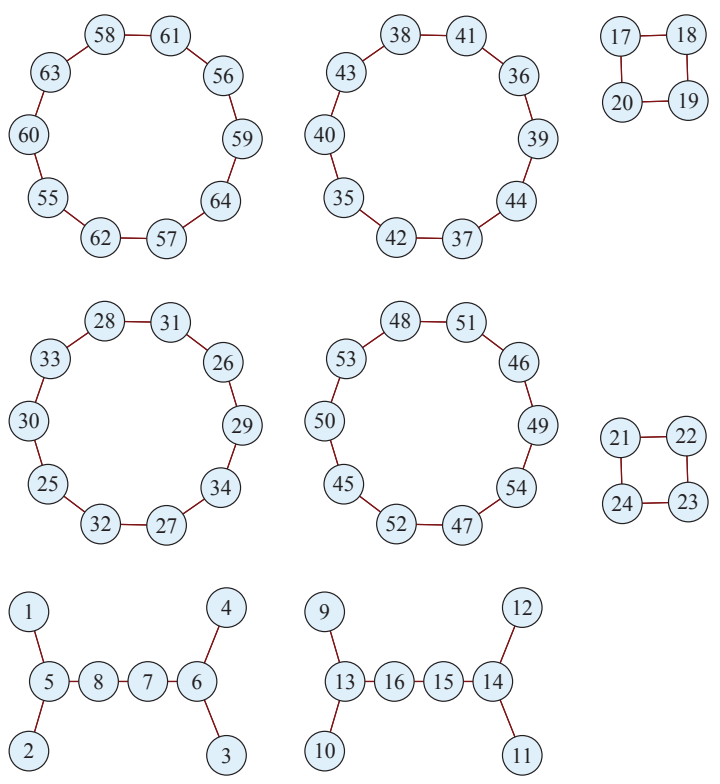

Figure 4. Fundamental fusion graph of $N_{5}$ in the Drinfeld double of the binary dihedral $\widehat{D}_{5}$.

The first $n+3$ irreps of the Drinfeld double of the binary dihedral group $\widehat{D}_{n}$ are classical (they can be identified with the irreps of $\widehat{D}_{n}$ ). They are of dimensions 1 or 2 . Their square sum is $4 n$. Among them, $n-1$ are of dimension 2 (the others are of dimension 1). We draw below (Fig. 1) the fusion graphs associated with these irreps of dimension 2.

$\widehat{D}_{2}: N_{c}=5,5,4,4,4$. Quantum dimensions: $\left(1_{4}, 2_{1} ; 1_{4}, 2_{1} ; 2_{4} ; 2_{4} ; 2_{4}\right)$.

$\widehat{D}_{3}: N_{c}=6,6,6,4,4,6$. Quantum dimensions: $\left(1_{4}, 2_{2} ; 1_{4}, 2_{2} ; 2_{6} ; 3_{4} ; 3_{4} ; 2_{6}\right)$.

$\widehat{D}_{4}: N_{c}=7,7,8,4,4,8,8$. Quantum dimensions: $\left(1_{4}, 2_{3} ; 1_{4}, 2_{3} ; 2_{8} ; 4_{4} ; 4_{4} ; 2_{8} ; 2_{8}\right)$

$\widehat{D}_{5}: N_{c}=8,8,4,4,10,10,10,10$. Quantum dimensions: $\left(1_{4}, 2_{4} ; 1_{4}, 2_{4} ; 5_{4} ; 5_{4} ; 2_{10} ; 2_{10} ; 2_{10} ; 2_{10}\right)$.

We analyse the case of $\widehat{D}_{5}$, the other tested cases are similar. The 12 irreps labeled 3,4 , 11, 12, 17, 18, 19, 20, 21, 22, 23, 24 on Fig. 4 are complex. The others $(64-12=52)$ are self-conjugate, with 28 being real and 24 quaternionic. The sum rule (7) holds. Note: The sum $\Sigma_{j}$ vanishes for 50 irreps: the 12 irreps that are complex, but also for 38 others, that are self-conjugate, namely all the 24 quaternionic and 14 real. On the other hand $\Sigma_{j}$ does not vanish on the 14 real irreps $1,2,6,8,25,27,29,31,33,35,37,39,41,43$. All these vanishing $\Sigma$ follow from the existence of some unit, as in Proposition 3. Thus there are no accidental cancellation in that case. See Table 1 for a summary. 
Table 1. Data and status of the sumrules (7) and (8) for Drinfeld doubles of some subgroups of SU(2). In each box, $n \checkmark$ means the number of irreps which satisfy the sumrule in question, the sign $\checkmark$ alone meaning "all of them", $n A$ gives the number of "accidental" vanishings, not due to the existence of a unit as in Proposition 3.

\begin{tabular}{|c|c|c|c|c|c|c|c|}
\hline name & $\begin{array}{c}(7) \\
\text { before } \\
\text { doubling }\end{array}$ & $r$ & $\begin{array}{c}\# i, i \neq \bar{\imath}, \forall j \\
\sum_{k} N_{i j}^{k} \stackrel{?}{=} \sum_{k} N_{\bar{\imath} j}^{k}\end{array}$ & $\begin{array}{c}\# \text { complex } \\
\# \sum_{j} S_{i j}=0 \\
\end{array}$ & $\begin{array}{c}\# \text { quatern. } \\
\# \sum_{j} S_{i j}=0\end{array}$ & $\begin{array}{c}\# \text { real } \\
\# \sum_{j} S_{i j}=0\end{array}$ & \#units \\
\hline $\mathbb{Z}_{5}$ & $\checkmark$ & 25 & $\checkmark$ & $\begin{array}{c}24 \\
24 \checkmark 0 A\end{array}$ & 0 & $\begin{array}{l}1 \\
0\end{array}$ & 25 \\
\hline $\mathbb{Z}_{6}$ & $\checkmark$ & 36 & $\checkmark$ & $\begin{array}{c}32 \\
32 \checkmark 0 A \\
\end{array}$ & 0 & $\begin{array}{c}4 \\
3 \checkmark 0 A \\
\end{array}$ & 36 \\
\hline$\widehat{\bar{D}}_{2}$ & $\checkmark$ & 22 & $\checkmark$ & 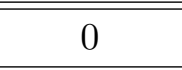 & $\begin{array}{c}8 \\
8 \checkmark^{0} 0 A \\
\end{array}$ & $\begin{array}{c}14 \\
6 \checkmark 0 A \\
\end{array}$ & 8 \\
\hline$\widehat{D}_{3}$ & $\checkmark$ & 32 & $\checkmark$ & $\begin{array}{c}12 \\
12 \checkmark 0 \mathrm{~A}\end{array}$ & $\begin{array}{c}8 \\
8 \checkmark^{0} 0 A\end{array}$ & $\begin{array}{l}12 \\
6 \checkmark 0 A\end{array}$ & 8 \\
\hline$\widehat{D}_{4}$ & $\checkmark$ & 46 & $\checkmark$ & 0 & $\begin{array}{c}20 \\
20 \checkmark 0 A\end{array}$ & $\begin{array}{c}26 \\
12 \checkmark 0 A\end{array}$ & 8 \\
\hline$\widehat{D}_{5}$ & $\checkmark$ & 64 & $\checkmark$ & $\begin{array}{c}12 \\
12 \checkmark 0 A \\
\end{array}$ & $\begin{array}{c}24 \\
24 \checkmark 0 A \\
\end{array}$ & $\begin{array}{c}28 \\
14 \checkmark 0 A\end{array}$ & 8 \\
\hline$\widehat{T}$ & $\checkmark$ & 42 & $\checkmark$ & $\begin{array}{c}32 \\
32 \checkmark 4 A \\
\end{array}$ & $\begin{array}{c}4 \\
4 \checkmark 0 A \\
\end{array}$ & $\begin{array}{c}6 \\
2 \sqrt{ } 2 A \\
\end{array}$ & 6 \\
\hline$\widehat{O}$ & $\checkmark$ & 56 & $\checkmark$ & 0 & $\begin{array}{c}26 \\
26 \checkmark \\
26 \mathrm{~V}\end{array}$ & $\begin{array}{c}30 \\
13 \checkmark 3 A\end{array}$ & 4 \\
\hline$\widehat{I}$ & $\checkmark$ & 74 & $\checkmark$ & 0 & $\begin{array}{c}36 \\
36 \checkmark 0 A\end{array}$ & $\begin{array}{c}38 \\
16 \checkmark 16 A\end{array}$ & 2 \\
\hline
\end{tabular}

\subsubsection{Drinfeld double of the binary tetrahedral}

Order of the group: 24

GAP nomenclature: SmallGroup(24,3). Alternate name: SL(2,3)

Class number: $\ell=7$

Rank: $r=42$

$N_{c}=7,7,6,6,4,6,6$

Quantum dimensions: $\left(1_{3}, 2_{3}, 3_{1} ; 1_{3}, 2_{3}, 3_{1} ; 4_{6} ; 4_{6} ; 6_{4} ; 4_{6} ; 4_{6}\right)$

$d_{\mathcal{B}}=2^{5} 3^{1} 13^{1} 599^{1}$

Embedding label: 4. See Fig. 5.
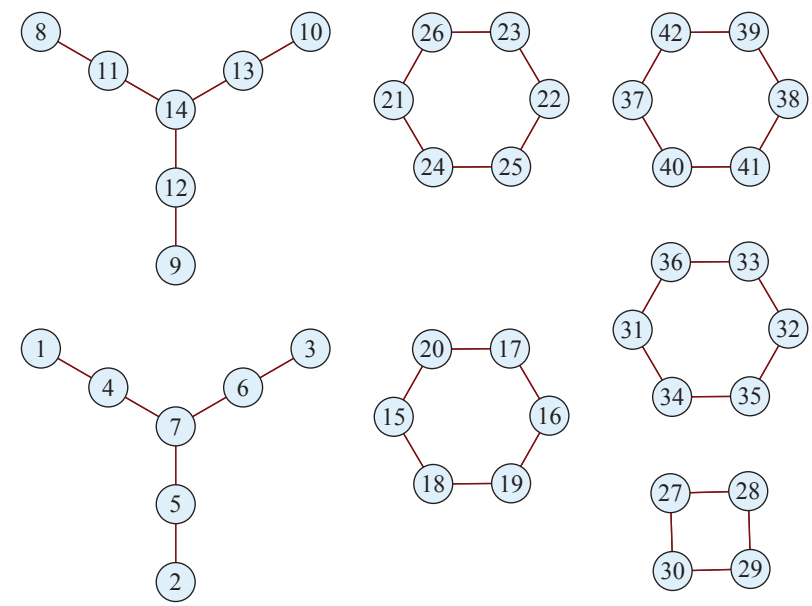

Figure 5. Fusion graph $N_{4}$ of the Drinfeld double of the binary tetrahedral.

The graph of $N_{4}$ is displayed on Fig. 5. There is a similar graph for the fusion matrix $N_{11}$. See Fig. 6 for a joint plot of both.

The 10 irreps labeled 1, 4, 7, 8, 11, 14, 27, 28, 29, 30 on Fig. 5 are self-conjugate. The others $(42-10=32)$ are complex. The sum rule (7) holds. 

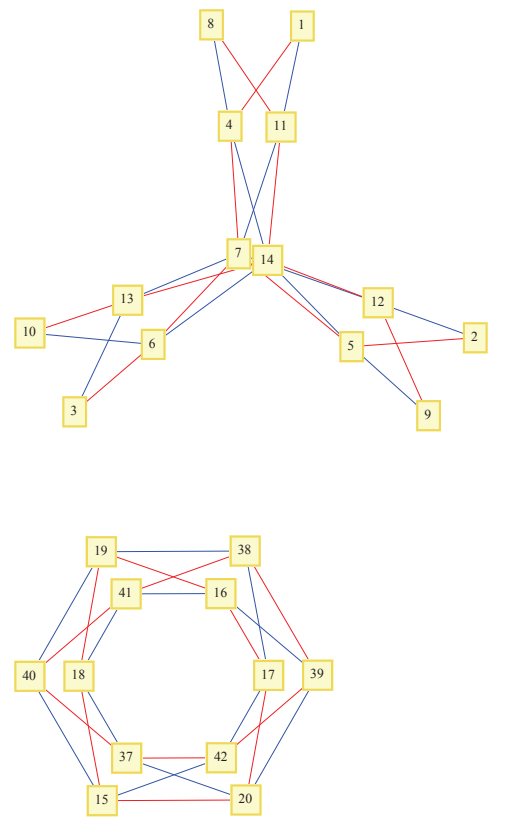
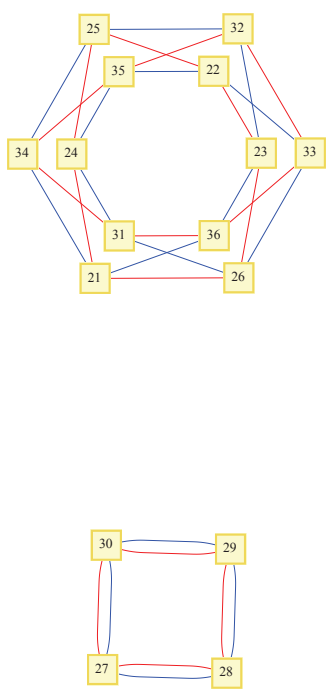

Figure 6. Fusion graphs $N_{4}$ and $N_{11}$ of the double of the binary tetrahedral (simultaneous plot).

Note. The sum $\Sigma_{j}$ vanishes for 38 irreps: the 32 that are complex, but also for 6 others, including 2 real and the 4 quaternionic. In other words $\Sigma_{j}$ does not vanish for the 4 real irreps $1,7,27,29$. In 4 complex and 2 real cases, the vanishing of $\Sigma$ is accidental.

\subsubsection{Drinfeld double of the binary octahedral}

Order of the group: 48

GAP nomenclature: $\operatorname{SmallGroup}(48,28)$

Class number: $\ell=8$

Rank: $r=56$

$N_{c}=8,8,6,8,4,6,8,8$

Quantum dimensions: $\left(1_{2}, 2_{3}, 3_{2}, 4_{1} ; 1_{2}, 2_{3}, 3_{2}, 4_{1} ; 8_{6} ; 66_{8} ; 12_{4} ; 8_{6} ; 6_{8} ; 6_{8}\right)$

$d_{\mathcal{B}}=2^{7} 37447^{1}$

Embedding labels: 4, 5. See Fig. 7 .

There is a similar graph for the fusion matrix $N_{12}$ (and also for $N_{5}$ and $N_{13}$ ). See Fig. 8 for a simultaneous plot of both $N_{4}$ and $N_{12}$.

The 56 irreps of the Drinfeld double are self-conjugate, 26 are quaternionic, 30 are real. The sum rule (7) holds trivially. Note: $\Sigma_{j}$ vanishes nevertheless for 39 irreps, all the quaternionic (26) and 13 real. In three real cases, the vanishing is accidental.

\subsubsection{Drinfeld double of the binary icosahedral}

Order of the group: 120

Class number: $\ell=9$

GAP nomenclature: SmallGroup(120,5). Alternate name: SL(2,5)

Rank: $r=74$

$N_{c}=9,9,6,4,10,10,6,10,10$

Quantum dimensions: $\left(1,2_{2}, 3_{2}, 4_{2}, 5,6 ; 1,2_{2}, 3_{2}, 4_{2}, 5,6 ; 20_{6} ; 30_{4} ; 12_{10} ; 12_{10} ; 20_{6} ; 12_{10} ; 12_{10}\right)$

$d_{\mathcal{B}}=2^{5} 61^{1} 89^{1} 263^{1}$

Embedding labels: 2 and 3. See Fig. 9. 

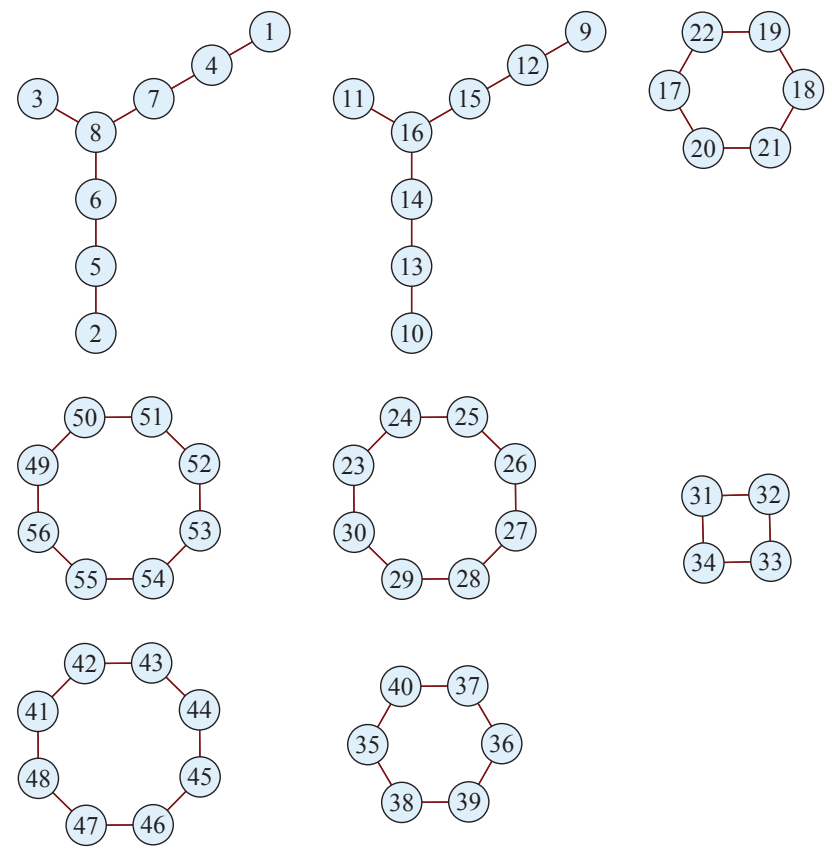

Figure 7. Fusion graph $N_{4}$ of the Drinfeld double of the binary octahedral.
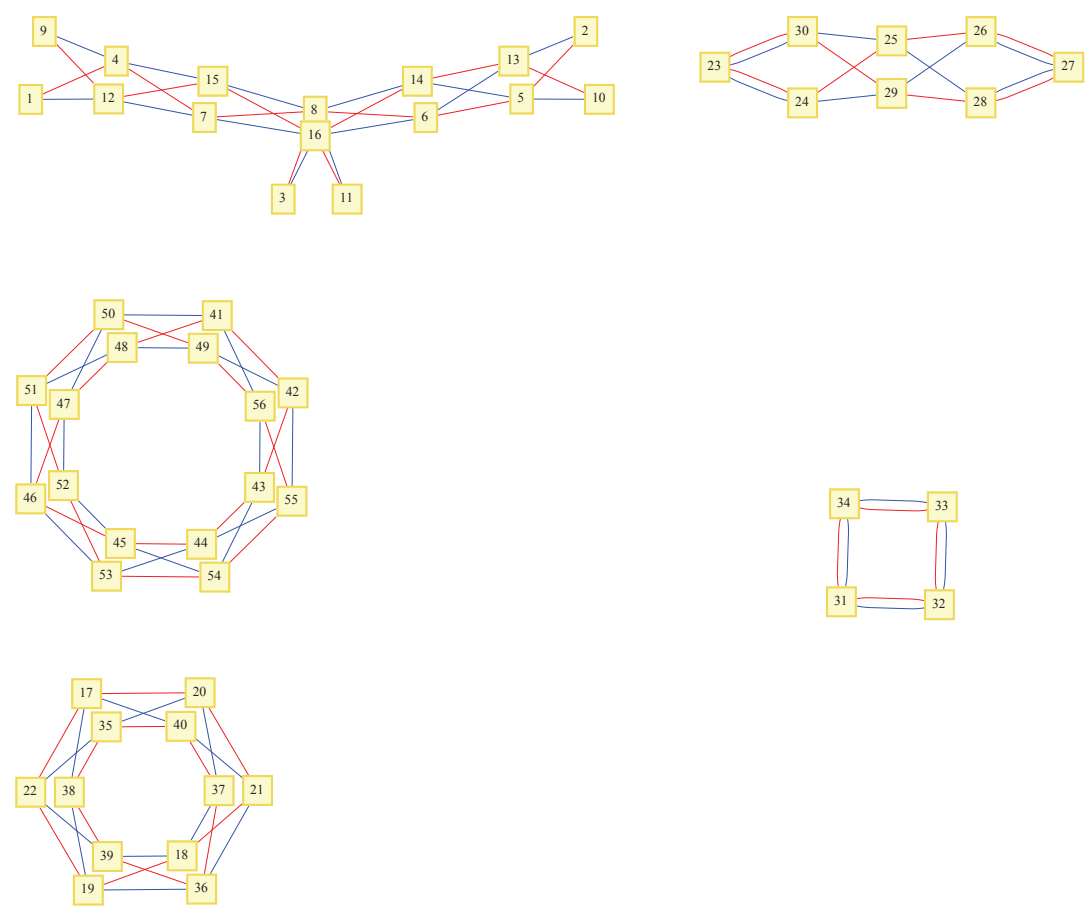

Figure 8. Fusion graphs $N_{4}$ and $N_{12}$ of the double of the binary octahedral (simultaneous plot).

There is a similar graph for the fusion matrix $N_{11}$ (and also for $N_{3}$ and $N_{12}$ ).

The 74 irreps of the Drinfeld double are self-conjugate: 38 are real, 36 are quaternionic. The sum rule (7) holds trivially. Note: $\Sigma_{j}$ vanishes nevertheless for 52 irreps, (16 real and all the 36 quaternionic ones). In the 16 real cases, the vanishing is accidental.

All the data concerning sumrules (7), (8) for doubles of subgroups of $\mathrm{SU}(2)$ have been gathered in Table 1. 

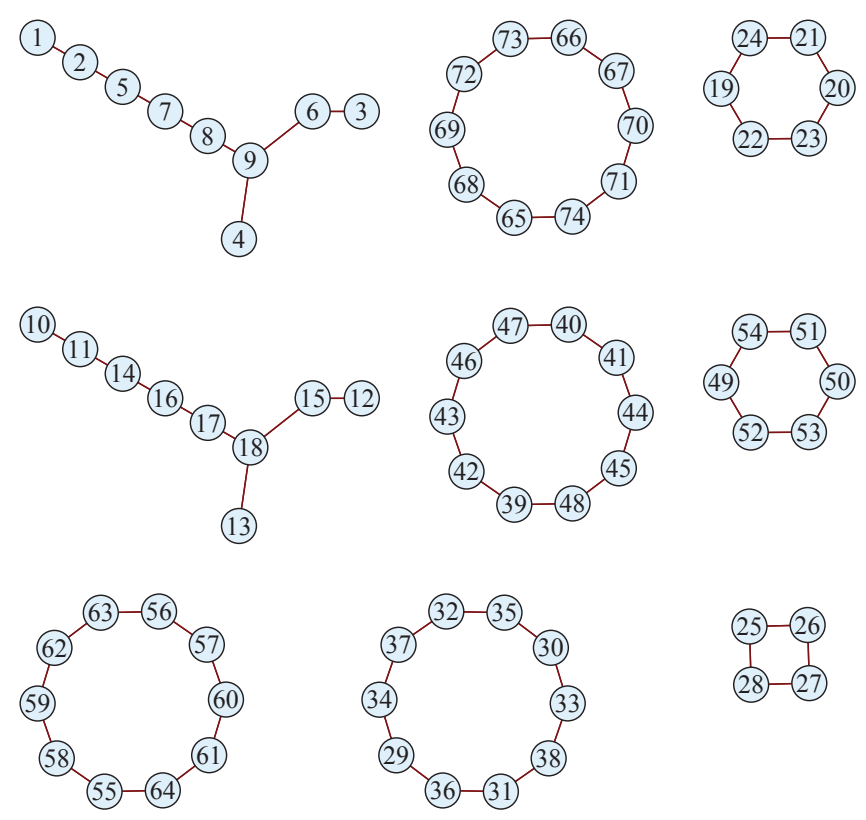

Figure 9. Fusion graph $N_{2}$ of the Drinfeld double of the binary icosahedral.
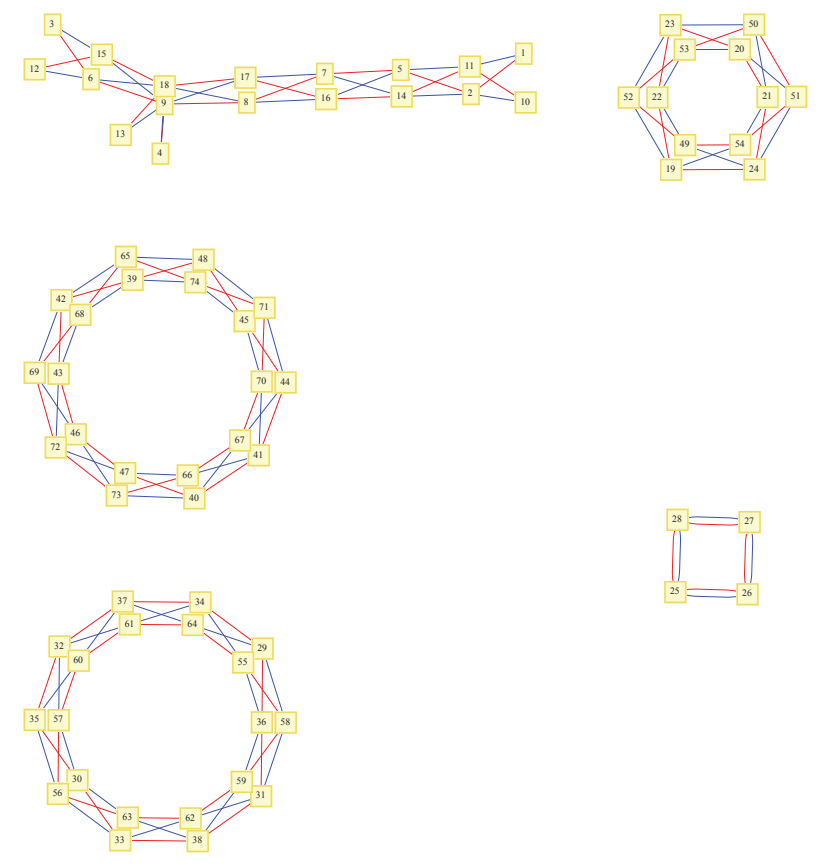

Figure 10. Fusion graphs $N_{2}$ and $N_{11}$ of the double of the binary icosahedral (simultaneous plot).

\subsection{Drinfeld doubles of finite subgroups of SU(3)}

\subsubsection{Remarks about finite subgroups of $\mathrm{SU}(3)$}

About the classification. The classification of the discrete finite groups of $\mathrm{SU}(3)$ is supposed to be well-known, since it goes back to 1917, see [2]. It has been however the object of some confusion in the more recent literature. We recall its main lines below and proceed to the analysis of several Drinfeld doubles. 
Historically, these subgroups were organized into twelve families ${ }^{11}$ : four series called $A, B$, $C, D$ and eight "exceptional" (precisely because they do not come into series) called $E, F, G$, $H, I, J$, and $H^{\star}, I^{\star}$. However, those called $H$ and $H^{\star}$ are not primitive, so that the primitive exceptional are only the six types $E, F, G, I, I^{\star}, J$ (or only five if one decides to forget about $I^{\star}$ ). Members of the types $A, B, C, D$ are never primitive: as described in [53], all the $A$, and some of the $B, C$ and $D$ are subgroups of a Lie subgroup of $\mathrm{SU}(3)$ isomorphic with $\mathrm{SU}(2)$, the other $B$ are in $\mathrm{U}(2) \sim(\mathrm{SU}(2) \times \mathrm{U}(1)) \mid \mathbb{Z}_{2}$ whereas the other $C$ and $D$, as well as $H$ and $H^{\star}$ are in a subgroup generated by $\mathrm{SO}(3) \subset \mathrm{SU}(3)$ and the center of $\mathrm{SU}(3)$. A construction of all these groups with explicit generators and relations can be found in [46]. Another way of organizing these subgroups (again into 12 families, but not quite the same) can be found in [29], and a new proposal, including only four general types, was recently made and described in [53]. A number of authors have entered the game in the past, or very recently, often with new notations and classifications, and presenting many explicit results that can be found in the following references (with hopefully not too many omissions) [18, 19, 21, 25, 34, 44, 52].

There is no apparent consensus about the way one should classify these subgroups, not to mention the notations to denote them (!), but everybody agrees about what they are, and in particular everybody agrees about the list of exceptional ones. We shall refrain from entering a dogmatic taxonomic discussion since our purpose is mostly to discuss a few examples of Drinfeld doubles associated with the finite subgroups of $\mathrm{SU}(3)$, and although we shall consider all the exceptional cases, we shall be happy with selecting only a few examples taken in the infinite series. Nevertheless, for the purpose of the description, we need to present our own notations.

We organize the list of subgroups of $\mathrm{SU}(3)$ as follows:

With notations of the previous section, we have the subgroups $\mathbb{Z}_{m} \times \mathbb{Z}_{n}$ and $\left\langle\mathbb{Z}_{m}, \widehat{D}_{n}\right\rangle,\left\langle\mathbb{Z}_{m}, \widehat{T}\right\rangle$, $\left\langle\mathbb{Z}_{m}, \widehat{O}\right\rangle,\left\langle\mathbb{Z}_{m}, \widehat{I}\right\rangle$ whose origin can be traced back to the fact that $\mathrm{U}(2) \sim(\mathrm{U}(1) \times \mathrm{SU}(2)) \mid \mathbb{Z}_{2}$ is a subgroup of $\mathrm{SU}(3)$. Here, by $\left\langle G_{1}, G_{2}\right\rangle$ we mean the group generated by the elements of the $\mathrm{SU}(3)$ subgroups $G_{1}$ and $G_{2}$ (this is not, in general, a direct product of $G_{1}$ and $G_{2}$ ). The orders of the above subgroups are respectively: $m \times n, m \times 2 n, m \times 24, m \times 48, m \times 120$ if $m$ is odd, and $m \times n, m / 2 \times 2 n, m / 2 \times 24, m / 2 \times 48, m / 2 \times 120$ if $m$ is even.

Then we have the ordinary dihedral $D_{n}$ (of order $2 n$ ) as well as the subgroups $\left\langle\mathbb{Z}_{m}, D_{n}\right\rangle$, of order $m \times 2 n$ if $m$ or $n$ is odd, and of order $m / 2 \times 2 n$ if $m$ and $n$ are both even.

We have two series of subgroups, called $\Delta_{3 n^{2}}=\left(\mathbb{Z}_{n} \times \mathbb{Z}_{n}\right) \rtimes \mathbb{Z}_{3}$, and $\Delta_{6 n^{2}}=\left(\mathbb{Z}_{n} \times \mathbb{Z}_{n}\right) \rtimes S_{3}$, for all positive integers $n$, that appear as $\mathrm{SU}(3)$ analogues of the binary dihedral groups (their orders appear as indices and the sign $\rtimes$ denotes a semi-direct product).

The $\Delta_{3 n^{2}}$ and $\Delta_{6 n^{2}}$ may themselves have subgroups that are not of that kind. For instance, for specific values of $p$ and $q$, with $p \neq q$, we have subgroups of the type $\left(\mathbb{Z}_{p} \times \mathbb{Z}_{q}\right) \rtimes \mathbb{Z}_{3}$ or $\left(\mathbb{Z}_{p} \times \mathbb{Z}_{q}\right) \rtimes S_{3}$, but we shall not discuss them further with the exception of Frobenius subgroups (see next entry) for the reason that such a digression would lie beyond the scope of our paper. Moreover the construction of a semi-direct product also involves a twisting morphism (that is not explicit in the previous notation) and, for this reason, it may happen that two groups built as semi-direct products from the same components are nevertheless non-isomorphic. The structure of the smallest SU(3) subgroups that cannot be written as direct product with cyclic groups, that do not belong to the previous types, that are neither exceptional (see below) nor of the Frobenius type (see next item) can be investigated from GAP. One finds $\left(\mathbb{Z}_{9} \times \mathbb{Z}_{3}\right) \rtimes \mathbb{Z}_{3}$, $\left(\mathbb{Z}_{14} \times \mathbb{Z}_{2}\right) \rtimes \mathbb{Z}_{3}, \mathbb{Z}_{49} \rtimes \mathbb{Z}_{3},\left(\mathbb{Z}_{26} \times \mathbb{Z}_{2}\right) \rtimes \mathbb{Z}_{3},\left(\mathbb{Z}_{21} \times \mathbb{Z}_{3}\right) \rtimes \mathbb{Z}_{3},\left(\mathbb{Z}_{38} \times \mathbb{Z}_{2}\right) \rtimes \mathbb{Z}_{3},\left(\mathbb{Z}_{91} \rtimes \mathbb{Z}_{3}\right)^{\prime}$, $\left(\mathbb{Z}_{91} \rtimes \mathbb{Z}_{3}\right)^{\prime \prime},\left(\mathbb{Z}_{18} \times \mathbb{Z}_{6}\right) \rtimes \mathbb{Z}_{3},\left(\mathbb{Z}_{28} \times \mathbb{Z}_{4}\right) \rtimes \mathbb{Z}_{3}, \ldots$, see the tables displayed in [44] or [33]. To illustrate a previous remark, notice that the above list includes two non-isomorphic subgroups of the type $\mathbb{Z}_{91} \rtimes \mathbb{Z}_{3}$, recognized as $\operatorname{SmallGroup}(273,3)$ and $\operatorname{SmallGroup}(273,4)$, that differ by the choice of the twisting morphism.

\footnotetext{
${ }^{11}$ Or, sometimes, into only 10 families, since $H^{\star} \sim H \times \mathbb{Z}_{3}$ and $I^{\star} \sim I \times \mathbb{Z}_{3}$ are trivially related to $H$ and $I$.
} 
The Frobenius subgroups. They are of the type $F_{3 m}=\mathbb{Z}_{m} \rtimes \mathbb{Z}_{3}$, but $m$ should be prime of the type $6 p+1$, i.e., $m=7,13,19,31, \ldots$ Their order is therefore $3 m=21,39,57,93, \ldots$ These subgroups are themselves subgroups of the $\Delta_{3 n^{2}}$ family, but they share common features and are somehow important for us. Indeed we checked that the property (7) fails systematically for them, and (7), (8) fails systematically for their Drinfeld double. We shall explicitly describe the Drinfeld double of $F_{21}$. The latter group was recently used for particle physics phenomenological purposes in [35].

The above subgroups exhaust the infinite series $A, B, C, D$ of [2], for instance the abelian subgroups $\mathbb{Z}_{m} \times \mathbb{Z}_{n}$ correspond to the diagonal matrices (the A type), but we prefer to describe explicitly a subgroup by its structure, for instance such as it is given by GAP, so that obtaining a direct correspondence with groups defined in terms of generators and relations, an information that we did not recall anyway, is not expected to be obvious.

We are now left with the eight exceptional subgroups. We sort them by their order and call them $\Sigma_{60}, \Sigma_{36 \times 3}, \Sigma_{168}, \Sigma_{60} \times \mathbb{Z}_{3}, \Sigma_{72 \times 3}, \Sigma_{168} \times \mathbb{Z}_{3}, \Sigma_{216 \times 3}, \Sigma_{360 \times 3}$. They correspond respectively to the groups $H, E, I, H^{\star}, F, I^{\star}, G, J$, in this order, of the Blichfeldt classification. The structure of these groups will be recalled later (see in particular Table 2). Six of them are ternary covers of exceptional groups in $\mathrm{SU}(3) / \mathbb{Z}_{3}$ called $\Sigma_{36}, \Sigma_{60}, \Sigma_{72}, \Sigma_{168}, \Sigma_{216}$ and $\Sigma_{360}$. There is a subtlety: the subgroups $\Sigma_{60}$ and $\Sigma_{168}$ of $\mathrm{SU}(3) / \mathbb{Z}_{3}$ are also subgroups of SU(3); their ternary covers in $\mathrm{SU}(3)$ are isomorphic to direct products by $\mathbb{Z}_{3}$ (this explains our notation); the other ternary covers are not direct products by $\mathbb{Z}_{3}$. Thus both $\Sigma_{60}$ and $\Sigma_{168}$, together with $\Sigma_{60} \times \mathbb{Z}_{3}$ and $\Sigma_{168} \times \mathbb{Z}_{3}$, indeed appear in the final list. Remember that only six exceptional subgroups - not the same "six" as before - define primitive inclusions in SU(3), since $\Sigma_{60}$ (the icosahedral subgroup of $\mathrm{SO}(3)$ ) and $\Sigma_{60} \times \mathbb{Z}_{3}$ don't. Some people use the notation $\Sigma_{p}$, where $p$ is the order, to denote all these exceptional subgroups, but this notation blurs the above distinction, and moreover it becomes ambiguous for $p=216$ since $\Sigma_{216}$ (a subgroup of $\mathrm{SU}(3) / \mathbb{Z}_{3}$ ) is not isomorphic to $\Sigma_{72 \times 3}$ (a subgroup of $\mathrm{SU}(3)$ ).

Miscellaneous remarks. In the literature, one can find several families of generators for the above groups; very often these generators are given as elements of GL(3), of SL(3), or of U(3), not of SU(3). Such findings do not imply any contradiction with the above classification since the latter is only given up to isomorphism.

Because of the embeddings of Lie groups $\mathrm{SU}(2) \subset \mathrm{SU}(3)$ and $\mathrm{SO}(3) \subset \mathrm{SU}(3)$, all the finite subgroups of $\mathrm{SU}(2)$ are finite subgroups of $\mathrm{SU}(3)$ and all the finite subgroups of $\mathrm{SO}(3)$ are subgroups of $\mathrm{SU}(3)$ as well. In particular all polyhedral groups and all binary polyhedral groups are subgroups of $\mathrm{SU}(3)$. Using the fact that $T \cong \Delta\left(3 \times 2^{2}\right), O \cong \Delta\left(6 \times 2^{2}\right)$, and $I \cong A_{5} \cong \Sigma_{60}$, the reader can recognize all of them in the above list.

Several calculations whose results are given below rely on some group theoretical information (for instance the determination of conjugacy classes and centralisers) that was taken from the GAP smallgroup library.

We shall now review a certain number of finite subgroups of $\mathrm{SU}(3)$ and their Drinfeld double. Like in the case of $\mathrm{SU}(2)$, we list for each of them a certain of data, and display one of their embedding fusion graphs. These graphs become fairly involved for large subgroups, and we label their vertices only for the smallest groups, while for the larger ones, only the connected component of the identity representation is labelled. Data on the way sum rules (7) and (8) are or are not satisfied, and the numbers of "accidental" vanishings, are gathered in Table 3.

\subsubsection{Drinfeld double of $F_{21}=\mathbb{Z}_{7} \rtimes \mathbb{Z}_{3}$}

Order of the group: 21

GAP nomenclature: $F_{21}=\operatorname{SmallGroup}(21,1)$

Class number: $\ell=5$ 
Table 2. Extra information on some subgroups of SU(3). Here, the integers $n$ always refer to cyclic groups $\mathbb{Z}_{n}$, so that, for instance, $2 \times 2$ means $\mathbb{Z}_{2} \times \mathbb{Z}_{2}$. Unless specified otherwise, the chains of symbols in the second and penultimate columns should be left parenthesized; for instance $3 \times 3 \rtimes 3 \rtimes Q_{8} \rtimes 3$ means $\left.\left(\left(\left(\mathbb{Z}_{3} \times \mathbb{Z}_{3}\right) \rtimes \mathbb{Z}_{3}\right) \rtimes Q_{8}\right) \rtimes \mathbb{Z}_{3}\right)$. Here $Q_{8} \cong \operatorname{Dic}_{2} \cong \widehat{D}_{2}$ is the quaternion group. $A_{n}$ and $S_{n}$ are the alternating and symmetric groups. The "dot" in $3 . A_{6}$ denotes a triple cover of $A_{6} . Z(G)$ denotes the center of $G, G^{\prime}$ its commutator subgroup, and $G / G^{\prime}$ its abelianization. The integer $|Z(G)|\left|G / G^{\prime}\right|$ gives the number of units in the fusion $\operatorname{ring}$. $\operatorname{Aut}(G)$ is the group of automorphisms of $G$. Out $(G)$ is the quotient $\operatorname{Aut}(G) / \operatorname{Inn}(G)$, where $\operatorname{Inn}(G)$ is the group of inner automorphisms, isomorphic with $G / Z(G)$. Finally $M(G)$ is the Schur multiplier of $G$.

\begin{tabular}{|c||c||c|c|c|c|c|}
\hline name & structure & $Z(G)$ & $G / G^{\prime}$ & $\operatorname{Out}(G)$ & $\operatorname{Aut}(G)$ & $M(G)$ \\
\hline \hline$T=\Delta\left(3 \times 2^{2}\right)$ & $A_{4}$ & 1 & 3 & 2 & $S_{4}$ & 2 \\
\hline$O=\Delta\left(6 \times 2^{2}\right)$ & $S_{4}$ & 1 & 2 & 1 & $S_{4}$ & 2 \\
\hline$F_{21}$ & $7 \rtimes 3$ & 1 & 3 & 2 & $7 \rtimes 3 \rtimes 2$ & 1 \\
\hline \hline$I=\Sigma_{60}$ & $A_{5}$ & 1 & 1 & 2 & $S_{5}$ & 2 \\
\hline$\Sigma_{36 \times 3}$ & $3 \times 3 \rtimes 3 \rtimes 4$ & 3 & 4 & $2 \times 2$ & $3 \rtimes 3 \rtimes 8 \rtimes 2$ & 1 \\
\hline$\Sigma_{168}$ & $\mathrm{SL}(3,2)$ & 1 & 1 & 2 & $\mathrm{SL}(3,2) \rtimes 2$ & 2 \\
\hline$\Sigma_{60} \times \mathbb{Z}_{3}$ & $\mathrm{GL}(2,4)$ & 3 & 3 & $2 \times 2$ & $2 \times S_{5}$ & 2 \\
\hline$\Sigma_{72 \times 3}$ & $3 \times 3 \rtimes 3 \rtimes Q_{8}$ & 3 & $2 \times 2$ & $S_{3}$ & $3 \times 3 \rtimes Q_{8} \rtimes 3 \rtimes 2$ & 1 \\
\hline$\Sigma_{168} \times \mathbb{Z}_{3}$ & $3 \times \mathrm{SL}(3,2)$ & 3 & 3 & $2 \times 2$ & $2 \times(\mathrm{SL}(3,2) \rtimes 2)$ & 2 \\
\hline$\Sigma_{216 \times 3}$ & $3 \times 3 \rtimes 3 \rtimes Q_{8} \rtimes 3$ & 3 & 3 & 6 & $3 \times\left(3 \times 3 \rtimes Q_{8} \rtimes 3 \rtimes 2\right)$ & 1 \\
\hline$\Sigma_{360 \times 3}$ & $3 . A_{6}$ & 3 & 1 & $2 \times 2$ & $A_{6} \rtimes 2 \rtimes 2$ & 2 \\
\hline
\end{tabular}

Classical dimensions: $1,1,1,3,3$

Rank: $r=25$

$N_{c}=5,3,3,7,7$

Quantum dimensions: $\left(1_{3}, 3_{2} ; 7_{3} ; 7_{3} ; 3_{7} ; 3_{7}\right)$

$d_{\mathcal{B}}=5^{1} 11^{1} 23^{1} 137^{1}$

Embedding labels: 4 and 5; see Fig. 11 for the fusion graph of $N_{4}$.

\subsubsection{Drinfeld double of $\Sigma_{60}$}

Remember that $\Sigma_{60}$ is both a subgroup of $\mathrm{SU}(3) / \mathbb{Z}_{3}$ and a subgroup of $\mathrm{SU}(3)$. Also, recall that this group is isomorphic to the icosahedron group $I$ of Section 4.1.1. Thus the 5 classical irreps of its double identify with the zero- "bi-ality" irreps of the binary icosahedron group $\widehat{I}$.

Order of the group: 60

GAP nomenclature: SmallGroup $(60,5)$. Alternate names: $A_{5}, \mathrm{SL}(2,4), \mathrm{I}$ (Icosahedral).

Class number: $\ell=5$

Classical dimensions: $1,3,3,4,5$

Rank: $r=22$

$N_{c}=5,4,3,5,5$

Quantum dimensions: $\left(1,3_{2}, 4,5 ; 15_{4} ; 200_{3} ; 12_{5} ; 12_{5}\right)$

$d_{\mathcal{B}}=2^{1} 5^{1} 11^{1} 10853^{1}$

Embedding labels: 2 and 3; see Fig. 12 for the fusion graph of $N_{2}$.

All the representations of $\Sigma(60)$ and of its double are real. 

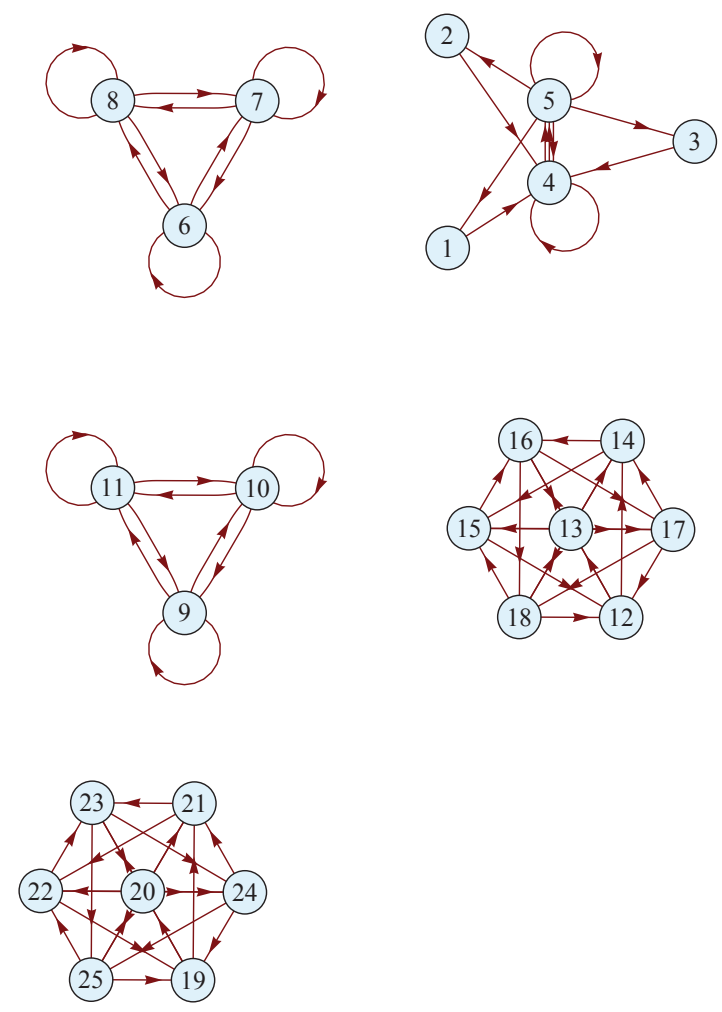

Figure 11. Fusion graph $N_{4}$ of the Drinfeld double of the group $F_{21}$.
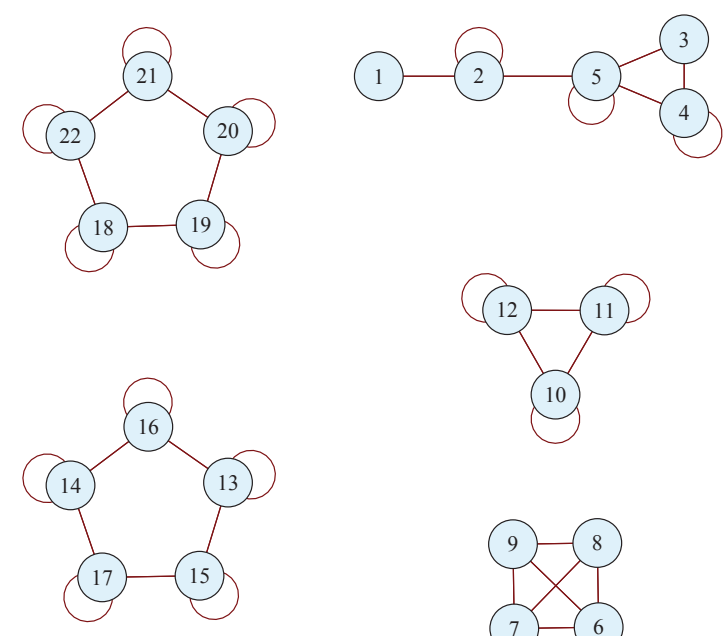

Figure 12. Fusion graph $N_{2}$ of the Drinfeld double of the group $\Sigma_{60}$.

\subsubsection{Drinfeld double of $\Sigma_{36 \times 3}$}

Order of the group: 108

GAP nomenclature: $\Sigma_{36 \times 3}=\operatorname{SmallGroup}(108,15)$

Class number: $\ell=14$

Classical dimensions: $1,1,1,1,3,3,3,3,3,3,3,3,4,4$

Rank: $r=168$

$N_{c}=14,12,14,14,9,9,12,12,12,12,12,12,12,12$

Quantum dimensions: $\left(1_{4}, 3_{8}, 4_{2} ; 9_{12} ; 1_{4}, 3_{8}, 4_{2} ; 1_{4}, 3_{8}, 4_{2} ; 12_{9} ; 12_{9} ; 9_{12} ; 9_{12} ; 9_{12} ; 9_{12} ; 9_{12}\right.$;

$$
\left.9_{12} ; 9_{12} ; 9_{12}\right)
$$


$d_{\mathcal{B}}=2^{3} 3^{5} 124477^{1}$

Embedding labels: 5 and 6; see Fig. 13 for the fusion graph $N_{5}$.

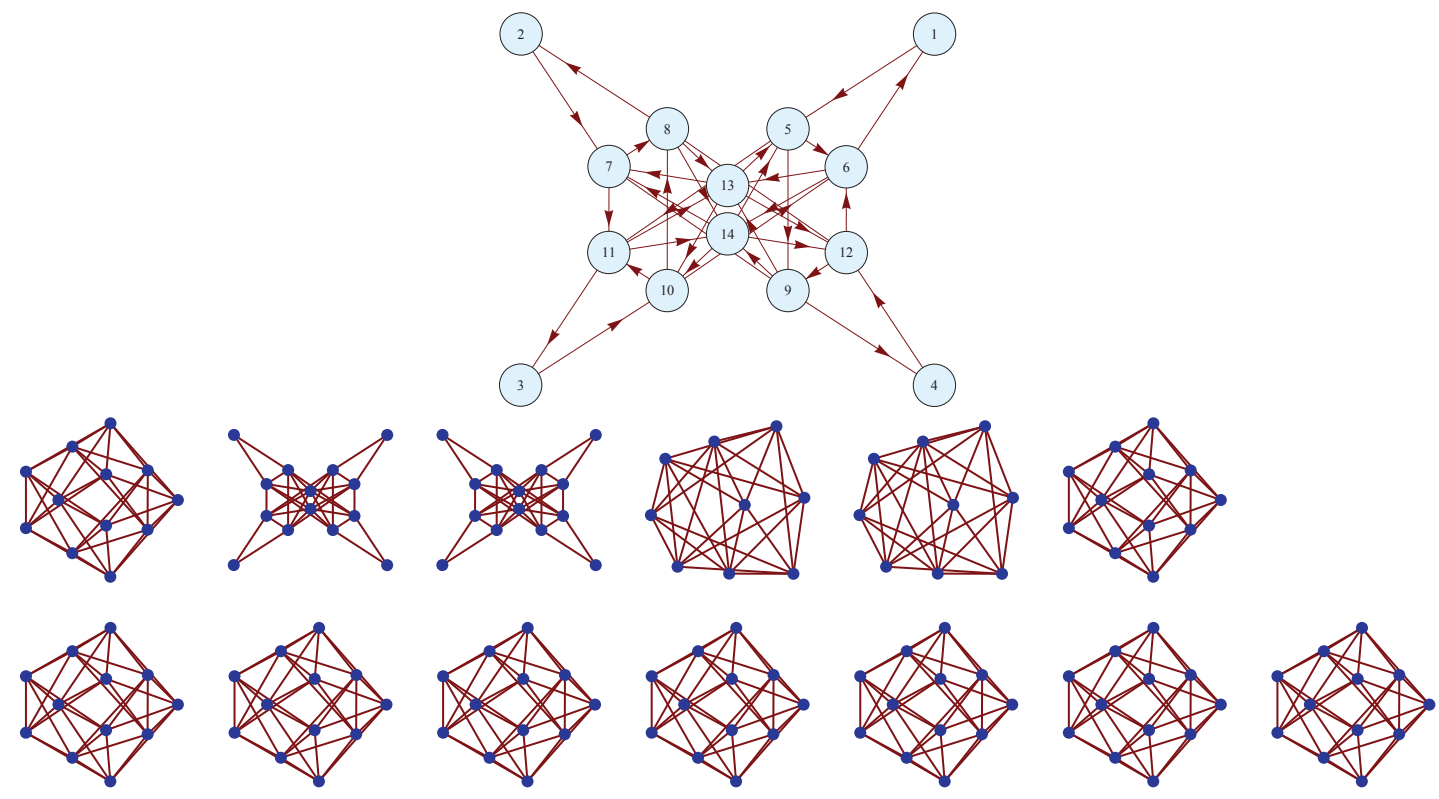

Figure 13. Fusion graph $N_{5}$ of the Drinfeld double of the group $\Sigma_{36 \times 3}$. Only the first connected component has been displayed with the labels of the vertices (irreps).

For illustration, let us analyze in some details the fusion graph of the quantum double of the group $G=\Sigma_{36 \times 3}$, see Fig. 13. The class number of $G$ is 14 . $G$ has nine 3-dimensional embedding representations labelled from 5 to 12. They all give fusion graphs sharing the same overall features. We have chosen to display $N_{5}$. The fusion graph of $G$ itself (the "classical graph") appears on the top. It is itself connected (it would not be so if we had chosen for instance $N_{13}$ or $N_{14}$, which correspond to 4-dimensional non faithful representations). It has 14 vertices. The fusion graph of the Drinfeld double $D(G)$ has 14 connected components. As the center of $G$ is $\mathbb{Z}_{3}$, the classical graph appears three times in the fusion graph of $D(G)$. The group $G$ has 14 conjugacy classes, but their stabilizers fall into only three types (up to isomorphisms): $G$ itself for the center (3 times), the cyclic group $\mathbb{Z}_{3} \times \mathbb{Z}_{3}$, that appears twice, and the cyclic group $\mathbb{Z}_{12}$, that appears nine times. The corresponding three kinds of connected components appear on Fig. 13. Apart from $G$ itself, these stabilizers are abelian, so that the number of their irreps (number of vertices of the corresponding connected components) are given by their order, respectively 9 and 12. The unity of the fusion ring (trivial representation) is labelled 1. This ring has twelve units. Four $(1,2,3,4)$, the "classical units", appear as the endpoints of the classical graph. The other eight $(4+4)$ are the corresponding endpoints on the two other copies of $G$. The units 1,2 are of real type, the units 3, 4 are of complex type (and actually conjugated). The eight embedding representations $5 \ldots 12$ are connected to the classical units. They appear in conjugate pairs $(5,6),(7,8)$ connected respectively to 1 and 2 , and $(9,10),(11,12)$ connected to 3 and 4 . Notice that a conjugated pair is attached to the same unit when this unit is real but to complex conjugate units when the unit is complex. Of course, the edges of the graph are oriented since 5 is not equivalent to its complex conjugate; the fusion graph $N_{6}$ can be obtained from the given graph, $N_{5}$, by reversing the arrows. The action of units clearly induces geometrical symmetries on the given fusion graph, nevertheless one expects that the fusion graphs associated with 5, 6, 7, 8 on the one hand, or with $9,10,11,12$ on the other, or with any of the corresponding vertices belonging to the three copies of the classical graph, although sharing the same overall features, will look slightly different, and the reader can check 
(for instance by drawing the graph $N_{9}$ ) that it is indeed so. (As a side remark, the classical graph of $\Sigma_{36 \times 3}$, drawn differently (see [12, Fig. 14]) leads after amputation of some vertices and edges to the fusion graph $\mathcal{E}^{(8)}$, the star-shaped exceptional module of $\mathrm{SU}(3)$ at level 5). The exponent of $G$ [9] is $m=12$, it is equal to the order of the modular matrix $T$, like for all Drinfeld doubles, and the entries of $S$ and $T$ (these are $168 \times 168$ matrices with entries labelled by the vertices of the Fig. 13) lie in the cyclotomic field $\mathbb{Q}(\xi)$ where $\xi=\exp (2 i \pi / m)$. We did not discuss Galois automorphisms in this paper, but let us mention nevertheless that there is also a Galois group acting by permutation on vertices, it is isomorphic to the multiplicative group $\mathbb{Z}_{m}^{\times}$of integers coprime to $m,[9,22]$.

\subsubsection{Drinfeld double of $\Sigma_{168}$}

Remember that $\Sigma_{168}$ is both a subgroup of $\mathrm{SU}(3) / \mathbb{Z}_{3}$ and a subgroup of SU(3). The group $\Sigma_{168}$ is the second smallest simple non-abelian group (the smallest being the usual icosahedral group $I \cong A_{5}$ of course!). It is often called the Klein group, or the smallest Hurwitz group.

GAP nomenclature: $\Sigma_{168}=\operatorname{SmallGroup}(168,42)$.

Alternate names: $\mathrm{SL}(3,2) \cong \operatorname{PSL}(3,2) \cong \mathrm{GL}(3,2) \cong \operatorname{PSL}(2,7)$.

Order of the group: 168

Class number: $\ell=6$

Classical dimensions: $1,3,3,6,7,8$

Rank: $r=32$

$N_{c}=6,5,3,4,7,7$

Quantum dimensions: $\left(1,3_{2}, 6,7,8 ; 21_{4}, 42 ; 56_{3} ; 42_{4} ; 24_{7} ; 24_{7}\right)$

$d_{\mathcal{B}}=2^{2} 4126561^{1}$

Embedding labels: 2 and 3. See Fig. 14 for the fusion graph $N_{2}$. The other embedding fusion graph $N_{3}$ is obtained from $N_{2}(14)$ by reversing the arrows.

For illustration, we shall give explicitly the $S$ matrix of this Drinfeld double in Appendix D.

\subsubsection{Drinfeld double of $\Sigma_{60} \times \mathbb{Z}_{3}$}

Order of the group: 180

GAP nomenclature: $\Sigma_{60} \times \mathbb{Z}_{3}=\operatorname{SmallGroup}(180,19)$.

Alternate names: $\mathrm{GL}(2,4)$.

Class number: $\ell=15$

Classical dimensions: $1,1,1,3,3,3,3,3,3,4,4,4,5,5,5$

Rank: $r=198$

$N_{c}=15,12,15,15,9,9,9,15,15,12,12,15,15,15,15$

Quantum dimensions: $\left(1_{3}, 3_{6}, 4_{3}, 5_{3} ; 15_{12} ; 1_{3}, 3_{6}, 4_{3}, 5_{3} ; 1_{3}, 3_{6}, 4_{3}, 5_{3} ; 20_{9} ; 20_{9} ; 20_{9} ; 12_{15} ; 12_{15}\right.$;

$d_{\mathcal{B}}=2^{1} 3^{6} 5^{1} 11^{1} 10853^{1}$

$\left.15_{12} ; 15_{12} ; 12_{15} ; 12_{15} ; 1215 ; 12_{15}\right)$

Embedding labels: 6 and its conjugate 9 , or 7 and its conjugate 8

See Fig. 15 for the fusion graph of $N_{6}$.

\subsubsection{Drinfeld double of $\Sigma_{72 \times 3}$}

Order of the group: 216

GAP nomenclature: $\Sigma_{72 \times 3}=\operatorname{SmallGroup}(216,88)$

Class number: $\ell=16$

Classical dimensions: $1,1,1,1,2,3,3,3,3,3,3,3,3,6,6,8$

Rank: $r=210$

$N_{c}=16,15,16,16,9,12,12,12,15,15,12,12,12,12,12,12$ 

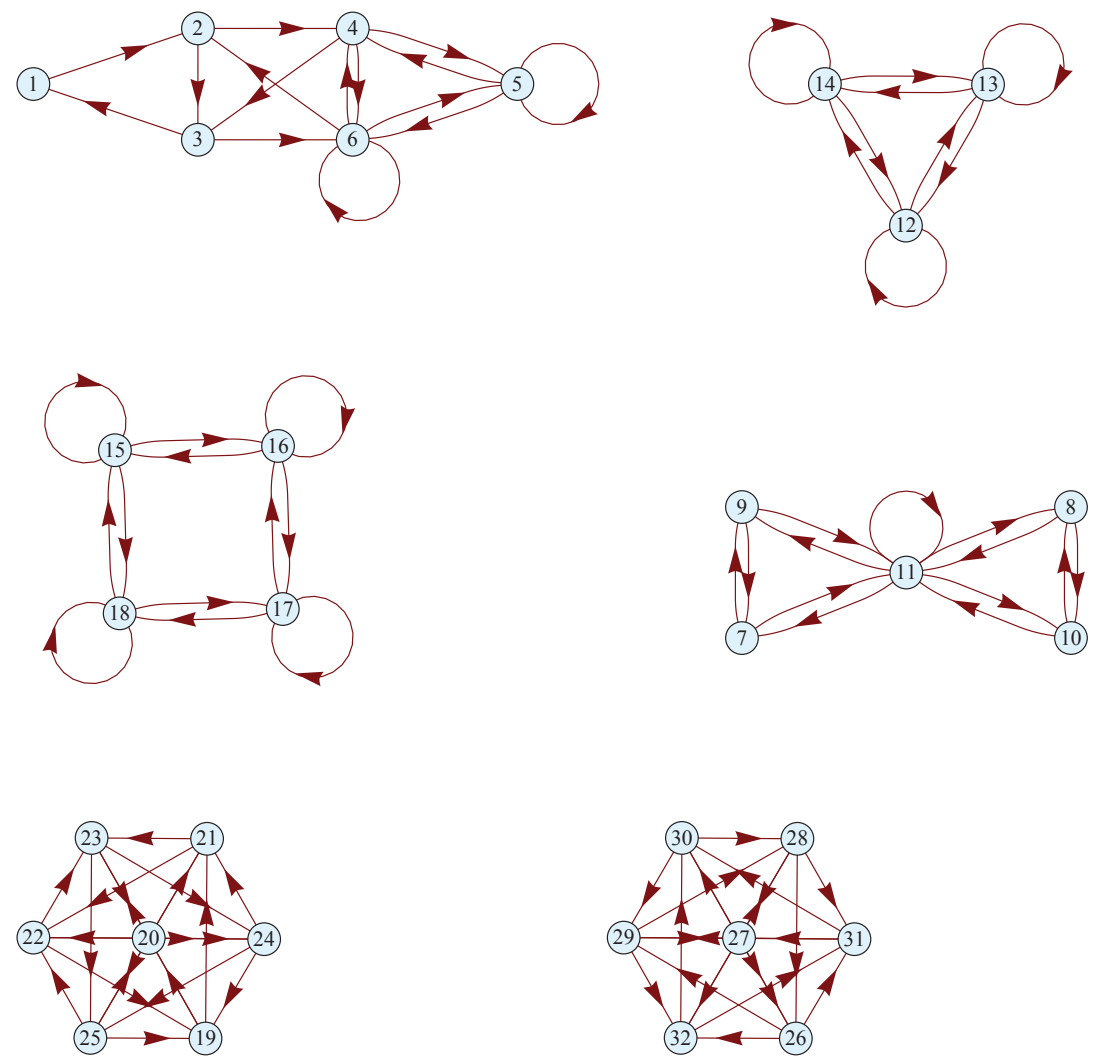

Figure 14. Fusion graph $N_{2}$ of the Drinfeld double of the Hurwitz group $\Sigma_{168}$.

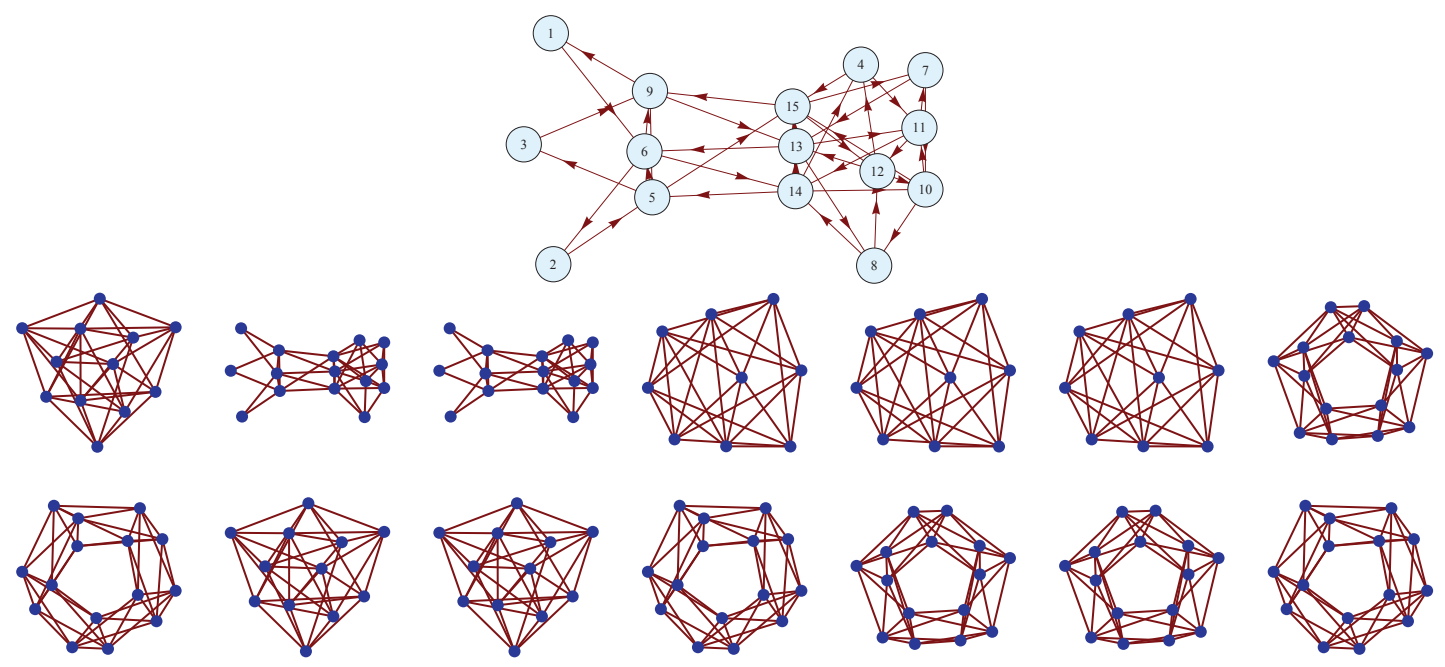

Figure 15. Fusion graph $N_{6}$ of the Drinfeld double of the group $\Sigma_{60} \times \mathbb{Z}_{3}$.

Quantum dimensions: $\left(1_{4}, 2,3_{8}, 6_{2}, 8 ; 9_{12}, 18_{3} ; 1_{4}, 2,3_{8}, 6_{2}, 8 ; 1_{4}, 2,3_{8}, 6_{2}, 8 ; 24_{9}\right.$;

$d_{\mathcal{B}}=2^{2} 3^{3} 23^{1} 59^{1} 8941^{1}$

$\left.18_{12} ; 18_{12} ; 18_{12} ; 9_{12}, 18_{3} ; 9_{12}, 18_{3} ; 18_{12} ; 18_{12} ; 18_{12} ; 18_{12} ; 18_{12} ; 18_{12}\right)$

Embedding labels: $6, \ldots, 13$.

\subsubsection{Drinfeld double of $\Sigma_{168} \times \mathbb{Z}_{3}$}

Order of the group: 504 

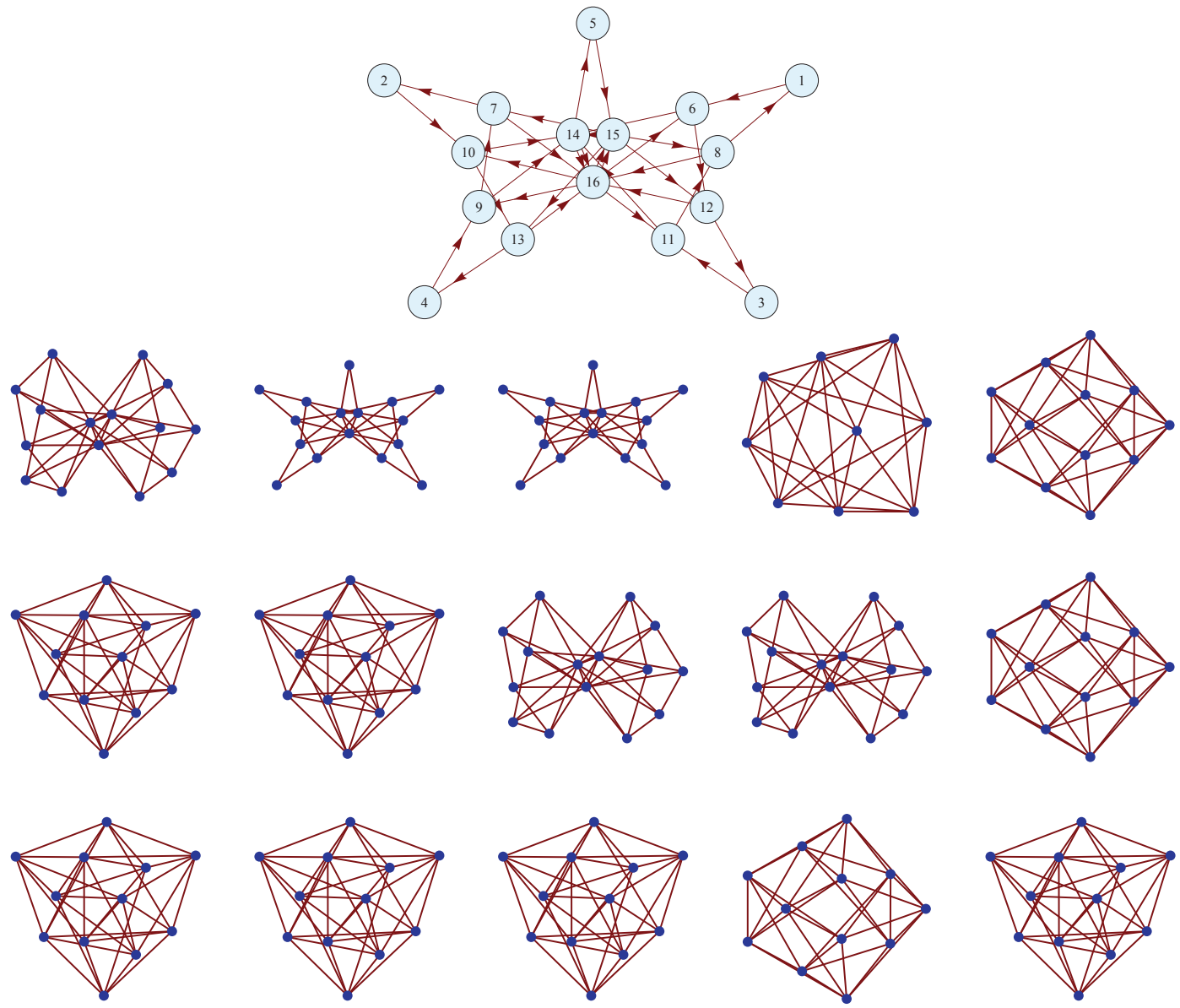

Figure 16. Fusion graph $N_{6}$ of the Drinfeld double of the group $\Sigma_{72 \times 3}$.

GAP nomenclature: $\Sigma_{168} \times \mathbb{Z}_{3}=\operatorname{SmallGroup}(504,157)$.

Class number: $\ell=18$

Classical dimensions: $1,1,1,3,3,3,3,3,3,6,6,6,7,7,7,8,8,8$

Rank: $r=288$

$N_{c}=18,15,18,18,9,9,9,12,15,15,21,21,12,12,21,21,21,21$

Quantum dimensions: $\left(1_{3}, 3_{6}, 6_{3}, 7_{3}, 8_{3} ; 21_{12}, 42_{3} ; 1_{3}, 3_{6}, 6_{3}, 7_{3}, 8_{3} ; 1_{3}, 3_{6}, 6_{3}, 7_{3}, 8_{3} ; 56_{9} ; 56_{9}\right.$;

$d_{\mathcal{B}}=2^{2} 3^{6} 4126561^{1}$

$$
\left.42_{12} ; 21_{12}, 42_{3} ; 21_{12}, 42_{3} ; 24_{21} ; 24_{21} ; 42_{12} ; 42_{12} ; 24_{21} ; 24_{21} ; 24_{21} ; 24_{21}\right)
$$

Embedding labels: 6, 7 and their conjugates 8, 9. See the graph of irrep 6 on Fig. 17.

\subsubsection{Drinfeld double of $\Sigma_{216 \times 3}$}

The group $\Sigma_{216 \times 3}$ is called the Hessian group, but this name also refers to $\Sigma_{216}$.

Order of the group: 648

GAP nomenclature: $\Sigma_{216} \times \mathbb{Z}_{3}=\operatorname{SmallGroup}(648,532)$

Class number: $\ell=24$

Classical dimensions: $1,1,1,2,2,2,3,3,3,3,3,3,3,6,6,6,6,6,6,8,8,8,9,9$

Rank: $r=486$

$N_{c}=\{24,21,24,24,27,9,9,12,21,21,27,27,27,27,27,27,12,12,18,18,18,18,18,18\}$

Quantum dimensions: $\left(1_{3}, 2_{3}, 3_{7}, 66_{6}, 8_{3}, 9_{2} ; 9_{9}, 18_{9}, 27_{3} ; 1_{3}, 2_{3}, 3_{7}, 6_{6}, 8_{3}, 9_{2} ; 1_{3}, 2_{3}, 3_{7}, 6_{6}, 8_{3}, 9_{2}\right.$;

$24_{27} ; 72_{9} ; 72_{9} ; 54_{12} ; 9_{9}, 18_{9}, 27_{3} ; 9_{9}, 18_{9}, 27_{3} ; 12_{18}, 24_{9} ; 12_{18}, 24_{9} ; 12_{18}, 24_{9}$;

$\left.12_{18}, 24_{9} ; 12_{18}, 24_{9} ; 12_{18}, 24_{9} ; 54_{12} ; 54_{12} ; 36_{18} ; 36_{18} ; 36_{18} ; 36_{18} ; 36_{18} ; 36_{18}\right)$ 


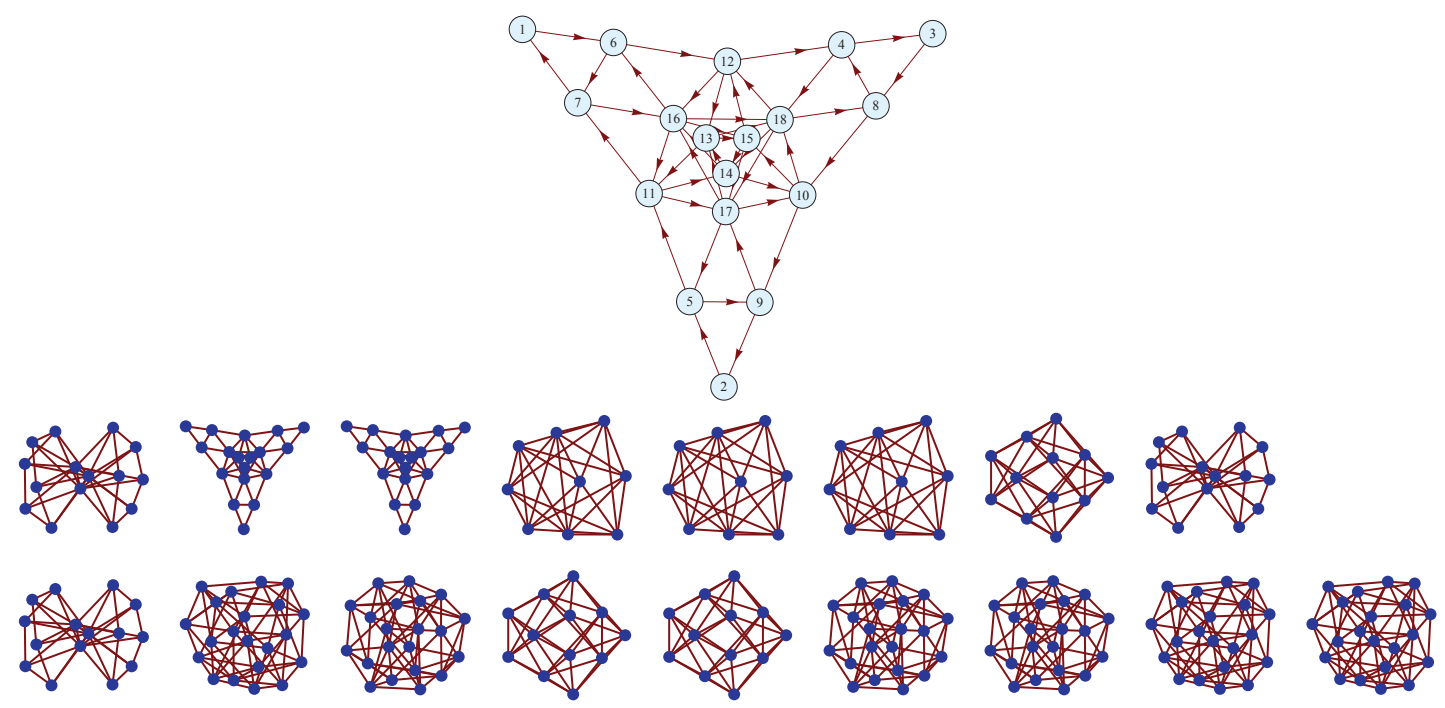

Figure 17. Fusion graph $N_{6}$ of the Drinfeld double of the group $\Sigma_{168} \times \mathbb{Z}_{3}$.

$d_{\mathcal{B}}=2^{2} 3^{6} 13^{1} 787^{1} 1481^{1}$

Embedding labels: (pairwise conjugates) 8, 10, 11 and 9, 13, 12.
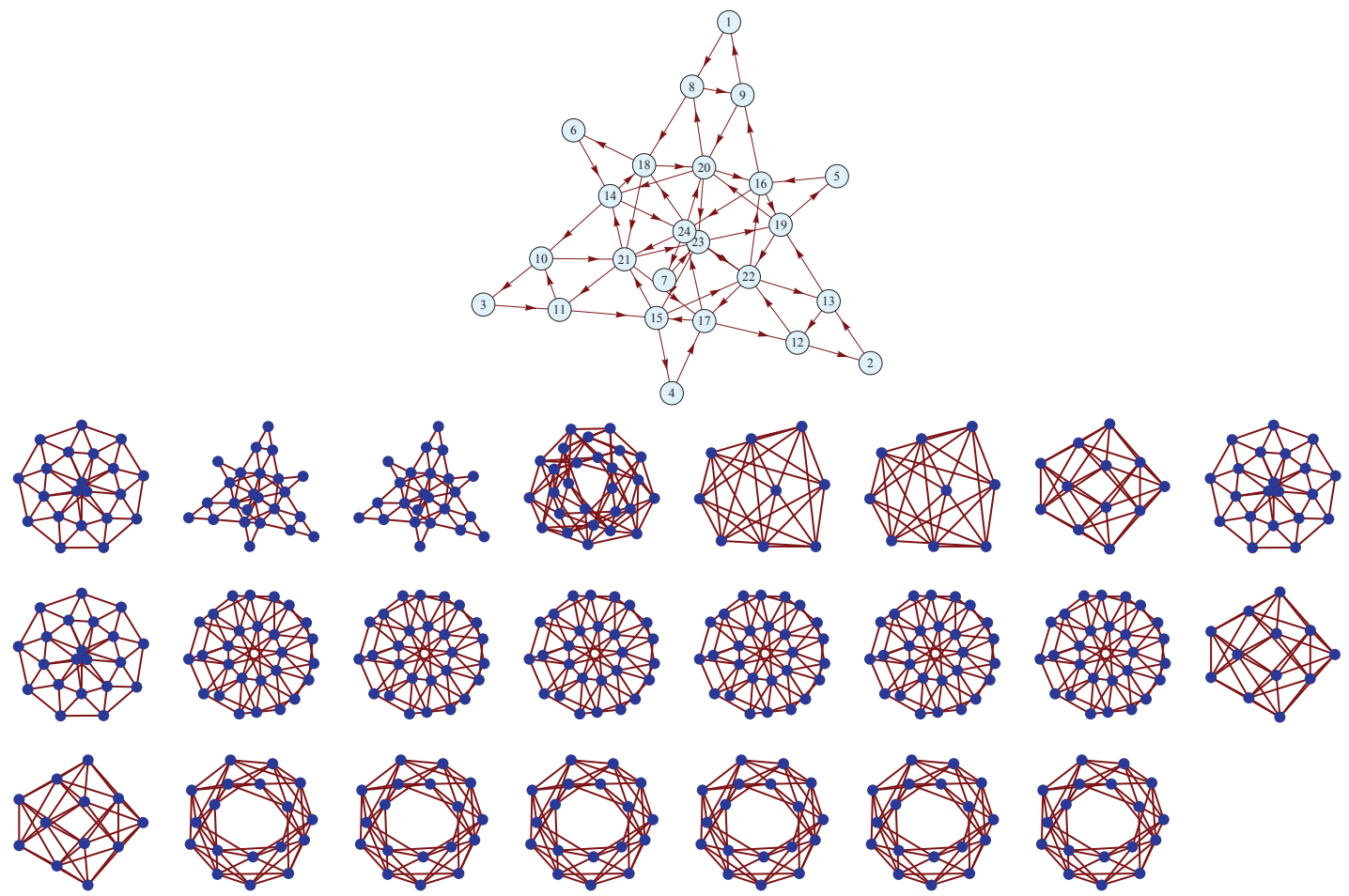

Figure 18. Fusion graph $N_{8}$ of the Drinfeld double of the group $\Sigma_{216 \times 3}$.

\subsubsection{Drinfeld double of $\Sigma_{360 \times 3}$}

The group $\Sigma_{360 \times 3}=\Sigma_{1080}$ (not always realized as a subgroup of SU(3)) is sometimes called the Valentiner group but this name also refers to $\Sigma_{360}$.

Order of the group: 1080

GAP nomenclature: $\Sigma_{360 \times 3}=\operatorname{SmallGroup}(1080,260)$. Alternate names: $3 . A_{6}$. 
Table 3. Data and status of the sumrules (7) and (8) for Drinfeld doubles of some subgroups of SU(3). The meaning of symbols is like in Table 1.

\begin{tabular}{|c|c|c|c|c|c|c|c|}
\hline name & $\begin{array}{c}(7) \\
\text { before } \\
\text { doubling }\end{array}$ & $r$ & $\begin{array}{c}\# i, i \neq i, \forall j \\
\sum_{k} N_{i j}^{k} \stackrel{?}{=} \sum_{k} N_{i j}^{k} \\
\end{array}$ & $\begin{array}{c}\# \text { complex } \\
\# \sum_{j} S_{i j}=0 \\
\end{array}$ & $\begin{array}{c}\# \text { quatern. } \\
\# \sum_{j} S_{i j}=0 \\
\end{array}$ & $\begin{array}{c}\# \text { real } \\
\# \sum_{j} S_{i j}=0\end{array}$ & \#units \\
\hline$\Delta_{3 \times 2^{2}}=T$ & $\checkmark$ & 14 & 2 & \begin{tabular}{c}
\multicolumn{8}{c}{} \\
$6 \checkmark^{0} 0 A$ \\
\end{tabular} & 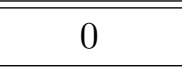 & $\begin{array}{l}6 \\
0 \\
\end{array}$ & 3 \\
\hline$\Delta_{6 \times 2^{2}}=O$ & $\checkmark$ & 21 & $\checkmark$ & 0 & 0 & $\begin{array}{c}21 \\
9 \checkmark 1 A\end{array}$ & 2 \\
\hline$F_{21}$ & $\times$ & 25 & 2 & $\begin{array}{c}24 \\
6 \checkmark 0 A \\
\end{array}$ & 0 & $\begin{array}{l}1 \\
0 \\
\end{array}$ & 3 \\
\hline$\Sigma_{60}=I$ & $\checkmark$ & 22 & $\bar{\checkmark}$ & 0 & 0 & $\begin{array}{c}22 \\
4 \checkmark \quad 0 A \\
\end{array}$ & 1 \\
\hline$\Sigma_{36 \times 3}$ & $\checkmark$ & 168 & $\checkmark$ & $\begin{array}{c}156 \\
156 \checkmark 14 A\end{array}$ & 0 & $\begin{array}{l}12 \\
3 \checkmark 1 A\end{array}$ & 12 \\
\hline$\Sigma_{168}$ & $\checkmark$ & 32 & 2 & $\begin{array}{c}16 \\
14 \checkmark 14 \mathrm{~A}\end{array}$ & 0 & $\begin{array}{c}16 \\
0\end{array}$ & 1 \\
\hline$\Sigma_{60} \times \mathbb{Z}_{3}$ & $\checkmark$ & 198 & 176 & $\begin{array}{c}176 \\
176 \checkmark 0 A\end{array}$ & 0 & $\begin{array}{c}22 \\
4 \checkmark 4 A\end{array}$ & 9 \\
\hline$\Sigma_{72 \times 3}$ & $x$ & 210 & 46 & $162 \checkmark^{184} 0 A$ & $\begin{array}{c}8 \\
6 \checkmark^{0} 0 A \\
\end{array}$ & $\begin{array}{c}18 \\
6 \checkmark 0 A\end{array}$ & 12 \\
\hline$\Sigma_{168} \times \mathbb{Z}_{3}$ & $\checkmark$ & 288 & 146 & $\begin{array}{c}272 \\
270 \checkmark 14 A\end{array}$ & 0 & $\begin{array}{c}16 \\
0 \\
\end{array}$ & 9 \\
\hline$\Sigma_{216 \times 3}$ & $\checkmark$ & 486 & 472 & $\begin{array}{c}472 \\
472 \checkmark 58 \mathrm{~A}\end{array}$ & $\begin{array}{c}4 \\
4 \checkmark \stackrel{4}{4} \checkmark\end{array}$ & $\begin{array}{c}10 \\
2 \checkmark 2 A \\
\end{array}$ & 9 \\
\hline$\Sigma_{360 \times 3}$ & $x$ & 240 & 52 & $\begin{array}{c}208 \\
176 \checkmark 20 A\end{array}$ & 0 & $\begin{array}{c}32 \\
0\end{array}$ & 3 \\
\hline
\end{tabular}

Class number: $\ell=17$

Classical dimensions: $1,3,3,3,3,5,5,6,6,8,8,9,9,9,10,15,15$

Rank: $r=240$

$N_{c}=17,15,17,17,9,9,12,15,15,15,15,12,12,15,15,15,15$

Quantum dimensions: $\left(1,3_{4}, 5_{2}, 6_{2}, 8_{2}, 9_{3}, 10,15_{2} ; 45_{12}, 90_{3} ; 1,3_{4}, 5_{2}, 6_{2}, 8_{2}, 9_{3}, 10,15_{2}\right.$; $1,3_{4}, 5_{2}, 6_{2}, 8_{2}, 9_{3}, 10,15_{2} ; 120_{9} ; 120_{9} ; 90_{12} ; 72_{15} ; 72_{15} ; 45_{12}, 90_{3}$;

$d_{\mathcal{B}}=2^{1} 3^{3} 734267099^{1}$

$\left.45_{12}, 90_{3} ; 90_{12} ; 90_{12} ; 72_{15} ; 72_{15} ; 72_{15} ; 72_{15}\right)$

Embedding labels: 2 and 4. See the graph of $N_{2}$ on Fig. 19.

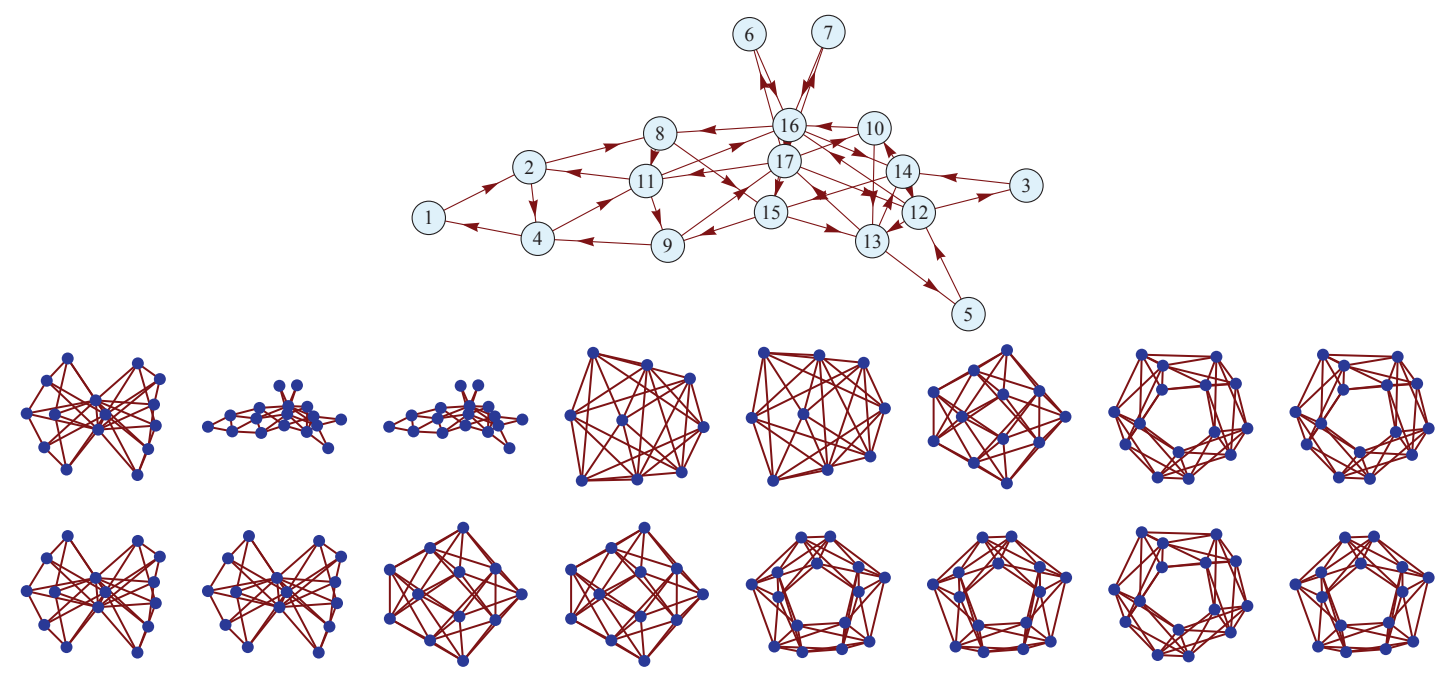

Figure 19. Fusion graph $N_{2}$ of the Drinfeld double of the group $\Sigma_{360 \times 3}$.

Note. All our results about these subgroups of $\mathrm{SU}(3)$, the violations of sumrules (7), (9) and (8) and the "accidental" vanishings have been collected in Table 3. 


\subsection{Drinfeld doubles of other finite groups (examples)}

We decided, in this paper, to discuss Drinfeld doubles of finite subgroups of $\mathrm{SU}(2)$ and of $\mathrm{SU}(3)$. But this overgroup plays no role and it could have been quite justified to organize our results in a different manner. Some finite subgroups of $\mathrm{SO}(3)$ have been already encountered as subgroups of $\mathrm{SU}(3)$, see above the cases of $T \cong \Delta_{3 \times 2^{2}}, O \cong \Delta_{6 \times 2^{2}}$ and $I \cong \Sigma_{60}$. We have also looked at the eight Mathieu groups, see for instance [26], $M_{9}, M_{10}, M_{11}, M_{12}, M_{21}, M_{22}, M_{23}, M_{24}$. Some $\left(M_{9}, M_{10}, M_{12}, M_{21}\right)$ satisfy the sumrule (7) before doubling, the others don't, and we were unable to understand the reason behind this.

\section{Conclusion and outlook}

Along this guided tour of Drinfeld doubles of finite subgroups of $\mathrm{SU}(2)$ and $\mathrm{SU}(3)$ we have put the emphasis on the discussion of the "sum rules" discovered in [8]: we have found that they are not generally satisfied, just like in the case of finite groups. In that respect the modularity of the fusion category of Drinfeld doubles is of no help. But we also found that, like in simple or affine Lie algebras, the two properties (7) and (8) are equivalent, in the sense that they are simultaneously satisfied for all $i$ and $j$ or not, a property that has no equivalent for finite groups, see Proposition 1. We found that certain conditions, like the existence of units, may grant the vanishing of some of these sum rules (Proposition 3). But a certain number of observed vanishing sum rules remains with no obvious explanation, whence the name "accidental" that we gave them. The bottom line is that at this stage, we have no precise criterion to decide for which group $G$ and for which irrep of $D(G)$ the sum rule (8) is satisfied. Clearly these points should deserve more attention.

The paper contains many figures. One of the curious properties of the fusion graph of an "embedding" representation of $D(G)$ - the appropriate generalization of the concept of fundamental faithful representation in this context - is that it contains as many connected components as there are irreps or classes in $G$.

Finally we want to mention another open issue that was not explicitly discussed in the present paper. It turns out that fusion graphs of doubles of finite subgroups of $\mathrm{SU}(2)$ have lots of similarities with those appearing in the discusion of rational $c=1$ conformal field theories obtained by orbifolding $\mathrm{SU}(2)$ by these finite subgroups [3, 14, 24, 49]. The precise relation, however, remains elusive. We hope to return to this point in the future.

\section{A About representations, faithfulness, and embeddings}

As usual, if no further indication is given, by "representation of a finite group" we mean "linear representation in a complex vector space". A representation of dimension (or degree) $n$, and kernel $K$, of the (abstract) finite group $G$, is in particular a morphism from $G$ to $\operatorname{GL}(n, \mathbb{C})$ and determines a concrete realization of the group $G \mid K$ (namely the image of this morphism) as a subgroup of $\mathrm{GL}(n, \mathbb{C})$. Conversely, if we choose some concrete finite subgroup of $\operatorname{GL}(n, \mathbb{C})$, and if this subgroup is isomorphic to $G$, the choice determines a faithful representation of $G$ (whose kernel $K$ is trivial) of dimension $n$. We shall say that a faithful $n$-dimensional representation of $G$ determines an embedding of $G$ into $\operatorname{GL}(n, \mathbb{C})$ and that it determines an irreducible embedding if the representation is both irreducible and faithful.

As usual, the table of characters of a finite group describes the evaluation of irreducible characters (listed vertically) on conjugacy classes (listed horizontally). The lack of faithfulness of an (irreducible) representation $\rho$ of dimension $n$ can be detected on the table of characters by the existence, along the line $\rho$, of more than one entry equal to $n$; this is a necessary and sufficient condition, as one may easily convince oneself. The representations appearing in a table of char- 
acters are irreducible and inequivalent (by definition of a table of characters), but a table may contain several faithful representations of the same dimension. Such representations therefore define subgroups of $\mathrm{U}(n)$ that are isomorphic to the given finite group, but are not conjugated.

As every representation of a finite group is equivalent to a unitary representation, we may consider that a faithful $n$-dimensional representation of $G$ determines an embedding of $G$ as a subgroup of $\mathrm{U}(n)=\mathrm{U}(n, \mathbb{C})$. Using the determinant homomorphism det from $\mathrm{U}(n)$ to $\mathrm{U}(1)$, one can consider $\mathrm{U}(n)$ as a semi-direct product of $\mathrm{U}(1)$ and $\mathrm{SU}(n)$. From any faithful unitary representation $\rho$ of the finite group $G$, i.e., from any embedding of $G$ into $\mathrm{U}(n)$, one obtains, by composing with the determinant, a $\mathrm{U}(1)$-valued representation, which may be trivial or not. In order to obtain an embedding of $G$ into $\mathrm{SU}(n)$ one needs the determinant to be equal to 1 on $\rho(G)$. Calculating the value of det for the chosen representation is not immediate from the table of characters alone, so that one usually needs to make use of an explicit set of representatives for the group generators.

An irreducible representation of $G$ (in a complex vector space) can be of real, complex, or quaternionic type. If it is faithful and volume preserving, it determines an embedding of $G$ into $\mathrm{SU}(n)$, but if it is furthermore of real type, one can obtain an embedding of $G$ into $\mathrm{SO}(n) \subset \mathrm{SU}(n)$, and if it is of quaternionic type (the dimension $n$ should be even), it determines an embedding into the compact group $\operatorname{Sp}(n / 2)=\mathrm{U}(n / 2, \mathbb{H})) \subset \mathrm{SU}(n)$. Remember that $\mathrm{Sp}(1)=\mathrm{SU}(2)$. The type of an irreducible representation is determined by the FrobeniusSchur indicator (not to be confused with the Schur index), which is +1 for $\mathbb{R}, 0$ for $\mathbb{C}$ and -1 for $\mathbb{H}$. In the cases like the Drinfeld doubles in which we have $S$ and $T$ matrices, see [8] for an expression of that Frobenius-Schur indicator.

\section{B About primitive and imprimitive finite subgroups of Lie groups}

A finite subgroup $G$ of a Lie group is called Lie primitive (we shall just write "primitive") if it is not included in a proper closed Lie subgroup (i.e., not a discrete subgroup) of that Lie group. One also says that $G$ is, or determines, a primitive inclusion in the chosen Lie group. If $G$ is not primitive, it is called imprimitive. A notion of Lie primitivity also exists for continuous subgroups of Lie groups but we do not need it. For instance the finite subgroups in $\mathrm{SO}(3)$ are the cyclic and dihedral groups together with the three exceptional ${ }^{12}$ polyhedral groups $T=A_{4}$, $O=S_{4}$ and $I=A_{5}$, and they are primitive in $\mathrm{SO}(3)$ except for the cyclic groups since the latter are subgroups $\mathrm{U}(1) \cong \mathrm{SO}(2) \subset \mathrm{SO}(3)$. The finite subgroups of $\mathrm{SU}(2)$ are the cyclic and binary dihedral groups, together with three exceptional: the binary polyhedral groups $\widehat{T}, \widehat{O}$ and $\widehat{I}$. The cyclic groups are not primitive in $\mathrm{SU}(2)$ since they are subgroups of $\mathrm{U}(1) \subset \mathrm{SU}(2)$, but they are not irreducible either as subgroups of $\mathrm{SU}(2)$ since the restriction to a cyclic group of its representation of complex dimension 2 splits as $1 \oplus 1$.

The situation is more subtle for subgroups of $\mathrm{SU}(3)$. For instance the existence of one (actually two) irreps of dimension 3 for the icosahedral group $\Sigma_{60}$ insures the existence of an irreducible embedding in $\mathrm{SU}(3)$, but these irreps of real type reflect the existence of the chain $\Sigma_{60} \subset \mathrm{SO}(3) \subset \mathrm{SU}(3)$. So, although irreducible, the embedding of $\Sigma_{60}$ in $\mathrm{SU}(3)$ is not primitive.

\section{About Schur covers}

Every projective representation of a finite group $G$, defined as a representation of $G$ on a projective space, can be lifted to an ordinary (i.e., linear) representation of another group called

\footnotetext{
${ }^{12}$ We call "exceptional" a subgroup of a given Lie group $\mathrm{SU}(n)$ that is not member of an infinite series.
} 
a Schur covering group after the seminal work of I. Schur at the beginning of the $20^{\text {th }}$ century (see [11] and references therein). Every finite group has a Schur cover but the latter may be non unique. As far as the classification of projective representations of $G$ is concerned, this non uniqueness does not matter because one can choose any Schur cover: the obtained projective representations of $G$ turn out to be the same. The case of alternating and symmetric groups is discussed in [28], see also [10].

We review a few basic facts about finite group extensions. An extension $K$ of $G$ by $N$ is defined by an onto group homomorphism $K \mapsto G$ with kernel $N$. The latter is a normal subgroup of $K$ and $G$ is isomorphic with $K \mid N$. The extension is called central whenever the kernel lies in the center $Z_{K}$ of $K$, in which case $N$ is abelian. A stem extension of $G$ is a central extension such that $N$ is not only contained in the center $Z_{K}$ of $K$ but also in the commutator subgroup $K^{\prime}$ of $K$, so $N \subset Z_{K} \cap K^{\prime}$. A Schur covering group (or a Schur cover) $K$ of $G$ can be abstractly defined as a stem extension of maximal order. Usually they are not split ${ }^{13}$ extensions ( $K$ has no reason to be a semi-direct product of $N$ and $G$ ).

Up to isomorphism, the normal subgroup $N$ defining the Schur extension does not depend on the choice of the chosen Schur covering group $K$ of $G$. More precisely, $N$ is isomorphic with what is called the Schur multiplier of $G$, defined as $M(G)=H_{2}(G, \mathbb{Z})=\left(H^{2}\left(G, \mathbb{C}^{\times}\right)\right)^{\star}$. Any projective representation of $G$ determines a 2-cocycle, and two projectively equivalent representations differ multiplicatively by a coboundary, so that projective representations of $G$ indeed fall into classes labelled by elements of $M(G)$. In particular, if the latter is trivial, the projective representations of $G$ are projectively equivalent to the linear ones.

Classifying Schur covers themselves, for a given $G$, is a difficult problem, and we shall not discuss it. Nevertheless it is worth mentioning that if $G$ is perfect (which means that it is equal to its commutator group $G^{\prime}$, or equivalently that its abelianization $H_{1}(G, \mathbb{Z})=G / G^{\prime}$ is trivial), then the Schur cover of $G$ is unique, up to isomorphism, and it may be called "universal cover", like in the theory of Lie groups. However, an imperfect group (like the group of the tetrahedron, see below) may have a unique Schur cover. Every non-abelian simple group is perfect.

Let us illustrate this discussion with subgroups of the Lie group $\mathrm{SO}(3)$. The universal cover of the latter is $\mathrm{SU}(2)$, and it is a binary cover, the kernel of the extension being $\mathbb{Z}_{2}$. The corresponding lifts, in $\mathrm{SU}(2)$, of the subgroups of $\mathrm{SO}(3)$ define the so-called binary groups, and the latter are indeed Schur covers of the former, when $M(G)$ is not trivial, but not all Schur covers of $\mathrm{SO}(3)$ subgroups lie in $\mathrm{SU}(2)$. The Schur multiplier of the exceptional polyhedral groups is $\mathbb{Z}_{2}$, and this means that they have two kinds of projective representations: those obtained from the reduction of the $\mathrm{SO}(3)$-representations (integral spin) and associated with their usual (linear) representations, but also the so-called binary representations (they are not linear representations) which can be obtained from the reduction of the $\mathrm{SU}(2)$ representations of half-integral spin. The tetrahedral group $T=A_{4}$ is not perfect, nevertheless it has a unique Schur cover ${ }^{14}, 2 . A_{4}$, that coincides with the binary tetrahedral group $\widehat{T}=\mathrm{SL}(2,3)$. The icosahedral group $I \sim A_{5} \sim \Sigma_{60}$ is the smallest non abelian simple group, and as such it is perfect (it is also the smallest non trivial perfect group). It has therefore only one Schur cover, the binary icosahedral group $\widehat{I}$, which is itself perfect, although non-simple, and even "superperfect" because $H_{1}(\widehat{I}, \mathbb{Z})=H_{2}(\widehat{I}, \mathbb{Z})=0$. However, the octahedral subgroup $O \sim S_{4}$ of $\mathrm{SO}(3)$ possesses two non-isomorphic Schur covers, one is the binary octahedral group $\widehat{O}$, a subgroup of $\mathrm{SU}(2)$, another is the group $\operatorname{GL}(2,3)$, which can be realized as a subgroup of $\mathrm{U}(2)$, but not of $\mathrm{SU}(2)$. Using GAP nomenclature $\widehat{O}$ can be recognized as SmallGroup $(48,28)$, whereas GL(2,3) is SmallGroup $(48,29)$. From the GAP table

\footnotetext{
${ }^{13}$ An extension is called split if $K$ is not only an extension, but also a semi-direct product of $N$ and $G$ with respect to some morphism $\phi$ from $G$ to the group $\operatorname{Aut}(N)$. One writes $K=N \rtimes_{\phi} G$, or just $K=N \rtimes G$, but $\phi$ is needed since the product is defined as $\left(n_{1}, g_{1}\right)\left(n_{2}, g_{2}\right)=\left(n_{1} \phi_{g_{1}}\left(n_{2}\right), g_{1} g_{2}\right)$. In that case both $N$ and $G$ are subgroups of $K$. A split extension can be central or not.

${ }^{14}$ A central extension of a group $G$ by the cyclic group $\mathbb{Z}_{k}$ is often denoted k.G.
} 
of characters only, we see that $\widehat{O}$ can be embedded into $\mathrm{SU}(2)$ because its 2 dimensional faithful irreducible representations are quaternionic (indeed $\mathrm{U}(1, \mathbb{H}) \sim \mathrm{SU}(2)$ ), whereas the 2 dimensional faithful irreducible representations of $\mathrm{GL}(2,3)$ are complex.

\section{The $S$ matrix of the Drinfeld double of the Klein group $\Sigma_{168}$}

The blocks of the $32 \times 32$ symmetric and unitary matrix $168 \times S(I, J)$, for $I=1, \ldots, 6, I \leq J \leq 6$ are given below. $(I, J)$ is a rectangular block, of dimension $u \times v$, with $u, v$ taken from the list $N c=(6,5,3,4,7,7)$ giving the orders of the centralizers for the conjugacy classes of $\Sigma_{168}$. We $\operatorname{set}^{15} \zeta=-\exp (i \pi / 7)$ and $\xi_{x_{0}, x_{1}, x_{2}, x_{3}, x_{4}, x_{5}}=x_{0}+x_{1} \xi+x_{2} \xi^{2}+x_{3} \xi^{3}+x_{4} \xi^{4}+x_{5} \xi^{5} \cdot \alpha_{1}=\xi_{0,0,0,0,1,2}$, $\alpha_{2}=\xi_{0,0,1,1,1,1}, \alpha_{3}=\xi_{0,0,2,1,0,0}, \alpha_{4}=\xi_{0,1,0,2,0,0}, \alpha_{5}=\xi_{0,1,1,0,1,0}, \alpha_{6}=\xi_{0,2,0,0,0,1}, \alpha_{7}=\xi_{1,0,0,1,1,0}$, $\alpha_{8}=\xi_{1,0,1,0,0,1}, \alpha_{9}=\xi_{1,1,1,0,1,0}, \alpha_{10}=\xi_{1,1,1,1,-1,1}, \alpha_{11}=\xi_{2,2,1,2,2,2}$. Then,

$I=1$,

$$
\begin{aligned}
& \left(\begin{array}{cccccc}
1 & 3 & 3 & 6 & 7 & 8 \\
3 & 9 & 9 & 18 & 21 & 24 \\
3 & 9 & 9 & 18 & 21 & 24 \\
6 & 18 & 18 & 36 & 42 & 48 \\
7 & 21 & 21 & 42 & 49 & 56 \\
8 & 24 & 24 & 48 & 56 & 64
\end{array}\right), \quad 21\left(\begin{array}{ccccc}
1 & 1 & 1 & 1 & 2 \\
-1 & -1 & -1 & -1 & -2 \\
-1 & -1 & -1 & -1 & -2 \\
2 & 2 & 2 & 2 & 4 \\
-1 & -1 & -1 & -1 & -2 \\
0 & 0 & 0 & 0 & 0
\end{array}\right), \quad 56\left(\begin{array}{cccccc}
1 & 1 & 1 \\
0 & 0 & 0 \\
0 & 0 & 0 \\
0 & 0 & 0 \\
1 & 1 & 1 \\
-1 & -1 & -1
\end{array}\right), \\
& 42\left(\begin{array}{cccc}
1 & 1 & 1 & 1 \\
1 & 1 & 1 & 1 \\
1 & 1 & 1 & 1 \\
0 & 0 & 0 & 0 \\
-1 & -1 & -1 & -1 \\
0 & 0 & 0 & 0
\end{array}\right), \quad 24\left(\begin{array}{cccccccc}
1 & 1 & 1 & 1 & 1 & 1 & 1 \\
\alpha 5 & \alpha 5 & \alpha 5 & \alpha 5 & \alpha 5 & \alpha 5 & \alpha 5 \\
-\alpha 9 & -\alpha 9 & -\alpha 9 & -\alpha 9 & -\alpha 9 & -\alpha 9 & -\alpha 9 \\
-1 & -1 & -1 & -1 & -1 & -1 & -1 \\
0 & 0 & 0 & 0 & 0 & 0 & 0 \\
1 & 1 & 1 & 1 & 1 & 1 & 1
\end{array}\right), \\
& 24\left(\begin{array}{ccccccccc}
1 & 1 & 1 & 1 & 1 & 1 & 1 \\
-\alpha 9 & -\alpha 9 & -\alpha 9 & -\alpha 9 & -\alpha 9 & -\alpha 9 & -\alpha 9 \\
\alpha 5 & \alpha 5 & \alpha 5 & \alpha 5 & \alpha 5 & \alpha 5 & \alpha 5 \\
-1 & -1 & -1 & -1 & -1 & -1 & -1 \\
0 & 0 & 0 & 0 & 0 & 0 & 0 \\
1 & 1 & 1 & 1 & 1 & 1 & 1
\end{array}\right),
\end{aligned}
$$

$I=2$,

$$
\begin{aligned}
& 21\left(\begin{array}{ccccc}
5 & 1 & -3 & 1 & -2 \\
1 & 5 & 1 & -3 & -2 \\
-3 & 1 & 5 & 1 & -2 \\
1 & -3 & 1 & 5 & -2 \\
-2 & -2 & -2 & -2 & 4
\end{array}\right), \quad\left(\begin{array}{lll}
0 & 0 & 0 \\
0 & 0 & 0 \\
0 & 0 & 0 \\
0 & 0 & 0 \\
0 & 0 & 0
\end{array}\right), \quad 21\left(\begin{array}{cccc}
2 & -2 & 2 & -2 \\
-2 & 2 & -2 & 2 \\
2 & -2 & 2 & -2 \\
-2 & 2 & -2 & 2 \\
0 & 0 & 0 & 0
\end{array}\right), \\
& \left(\begin{array}{lllllll}
0 & 0 & 0 & 0 & 0 & 0 & 0 \\
0 & 0 & 0 & 0 & 0 & 0 & 0 \\
0 & 0 & 0 & 0 & 0 & 0 & 0 \\
0 & 0 & 0 & 0 & 0 & 0 & 0 \\
0 & 0 & 0 & 0 & 0 & 0 & 0
\end{array}\right), \quad\left(\begin{array}{lllllll}
0 & 0 & 0 & 0 & 0 & 0 & 0 \\
0 & 0 & 0 & 0 & 0 & 0 & 0 \\
0 & 0 & 0 & 0 & 0 & 0 & 0 \\
0 & 0 & 0 & 0 & 0 & 0 & 0 \\
0 & 0 & 0 & 0 & 0 & 0 & 0
\end{array}\right)
\end{aligned}
$$

\footnotetext{
${ }^{15}$ The entries of $S$ lie in the cyclotomic field $\mathbb{Q}(\exp (2 i \pi / m))$ where $m$ is the exponent of the group [9]. Here $m=84$. There are many ways to express the same matrix elements.
} 
$I=3$,

$$
\begin{aligned}
& 56\left(\begin{array}{cccc}
2 & -1 & -1 \\
-1 & -1 & 2 \\
-1 & 2 & -1
\end{array}\right), \quad\left(\begin{array}{llll}
0 & 0 & 0 & 0 \\
0 & 0 & 0 & 0 \\
0 & 0 & 0 & 0
\end{array}\right), \quad\left(\begin{array}{lllllll}
0 & 0 & 0 & 0 & 0 & 0 & 0 \\
0 & 0 & 0 & 0 & 0 & 0 & 0 \\
0 & 0 & 0 & 0 & 0 & 0 & 0
\end{array}\right) \\
& \left(\begin{array}{lllllll}
0 & 0 & 0 & 0 & 0 & 0 & 0 \\
0 & 0 & 0 & 0 & 0 & 0 & 0 \\
0 & 0 & 0 & 0 & 0 & 0 & 0
\end{array}\right),
\end{aligned}
$$

$I=4$,

$$
84\left(\begin{array}{cccc}
1 & 0 & -1 & 0 \\
0 & -1 & 0 & 1 \\
-1 & 0 & 1 & 0 \\
0 & 1 & 0 & -1
\end{array}\right), \quad\left(\begin{array}{ccccccc}
0 & 0 & 0 & 0 & 0 & 0 & 0 \\
0 & 0 & 0 & 0 & 0 & 0 & 0 \\
0 & 0 & 0 & 0 & 0 & 0 & 0 \\
0 & 0 & 0 & 0 & 0 & 0 & 0
\end{array}\right),\left(\begin{array}{lllllll}
0 & 0 & 0 & 0 & 0 & 0 & 0 \\
0 & 0 & 0 & 0 & 0 & 0 & 0 \\
0 & 0 & 0 & 0 & 0 & 0 & 0 \\
0 & 0 & 0 & 0 & 0 & 0 & 0
\end{array}\right),
$$

$I=5$,

$$
\begin{array}{r}
24\left(\begin{array}{ccccccc}
3 & -\alpha_{9} & -\alpha_{9} & \alpha_{5} & -\alpha_{9} & \alpha_{5} & \alpha_{5} \\
-\alpha_{9} & \alpha_{3} & \alpha_{6} & -\alpha_{2} & -\alpha_{10} & \alpha_{8} & \alpha_{7} \\
-\alpha_{9} & \alpha_{6} & -\alpha_{10} & \alpha_{7} & \alpha_{3} & -\alpha_{2} & \alpha_{8} \\
\alpha_{5} & -\alpha_{2} & \alpha_{7} & -\alpha_{11} & \alpha_{8} & \alpha_{1} & \alpha_{4} \\
-\alpha_{9} & -\alpha_{10} & \alpha_{3} & \alpha_{8} & \alpha_{6} & \alpha_{7} & -\alpha_{2} \\
\alpha_{5} & \alpha_{8} & -\alpha_{2} & \alpha_{1} & \alpha_{7} & \alpha_{4} & -\alpha_{11} \\
\alpha_{5} & \alpha_{7} & \alpha_{8} & \alpha_{4} & -\alpha_{2} & -\alpha_{11} & \alpha_{1}
\end{array}\right), \\
24\left(\begin{array}{ccccccc}
3 & \alpha_{5} & \alpha_{5} & -\alpha_{9} & \alpha_{5} & -\alpha_{9} & -\alpha_{9} \\
\alpha_{5} & \alpha_{1} & -\alpha_{11} & -\alpha_{2} & \alpha_{4} & \alpha_{8} & \alpha_{7} \\
\alpha_{5} & -\alpha_{11} & \alpha_{4} & \alpha_{7} & \alpha_{1} & -\alpha_{2} & \alpha_{8} \\
-\alpha_{9} & -\alpha_{2} & \alpha_{7} & \alpha_{6} & \alpha_{8} & \alpha_{3} & -\alpha_{10} \\
\alpha_{5} & \alpha_{4} & \alpha_{1} & \alpha_{8} & -\alpha_{11} & \alpha_{7} & -\alpha_{2} \\
-\alpha_{9} & \alpha_{8} & -\alpha_{2} & \alpha_{3} & \alpha_{7} & -\alpha_{10} & \alpha_{6} \\
-\alpha_{9} & \alpha_{7} & \alpha_{8} & -\alpha_{10} & -\alpha_{2} & \alpha_{6} & \alpha_{3}
\end{array}\right),
\end{array}
$$

$I=6$,

$$
24\left(\begin{array}{ccccccc}
3 & -\alpha_{9} & -\alpha_{9} & \alpha_{5} & -\alpha_{9} & \alpha_{5} & \alpha_{5} \\
-\alpha_{9} & \alpha_{3} & \alpha_{6} & -\alpha_{2} & -\alpha_{10} & \alpha_{8} & \alpha_{7} \\
-\alpha_{9} & \alpha_{6} & -\alpha_{10} & \alpha_{7} & \alpha_{3} & -\alpha_{2} & \alpha_{8} \\
\alpha_{5} & -\alpha_{2} & \alpha_{7} & -\alpha_{11} & \alpha_{8} & \alpha_{1} & \alpha_{4} \\
-\alpha_{9} & -\alpha_{10} & \alpha_{3} & \alpha_{8} & \alpha_{6} & \alpha_{7} & -\alpha_{2} \\
\alpha_{5} & \alpha_{8} & -\alpha_{2} & \alpha_{1} & \alpha_{7} & \alpha_{4} & -\alpha_{11} \\
\alpha_{5} & \alpha_{7} & \alpha_{8} & \alpha_{4} & -\alpha_{2} & -\alpha_{11} & \alpha_{1}
\end{array}\right) .
$$

Klein's quartic

\section{References}

[1] Behrend R.E., Pearce P.A., Petkova V.B., Zuber J.-B., Boundary conditions in rational conformal field theories, Nuclear Phys. B 579 (2000), 707-773, hep-th/9908036.

[2] Blichfeldt H.F., Finite collineation groups, The University Chicago Press, Chicago, 1917.

[3] Cappelli A., D'Appollonio G., Boundary states of $c=1$ and $c=3 / 2$ rational conformal field theories, J. High Energy Phys. 2002 (2002), no. 2, 039, 45 pages, hep-th/0201173. 
[4] Cardy J.L., Boundary conditions, fusion rules and the Verlinde formula, Nuclear Phys. B 324 (1989), $581-596$.

[5] Cibils C., Tensor product of Hopf bimodules over a group, Proc. Amer. Math. Soc. 125 (1997), $1315-1321$.

[6] Coquereaux R., Character tables (modular data) for Drinfeld doubles of finite groups, PoS Proc. Sci. (2012), PoS(ICMP2012), 024, 8 pages, arXiv:1212.4010.

[7] Coquereaux R., Isasi E., Schieber G., Notes on TQFT wire models and coherence equations for SU(3) triangular cells, SIGMA 6 (2010), 099, 44 pages, arXiv:1007.0721.

[8] Coquereaux R., Zuber J.-B., On sums of tensor and fusion multiplicities, J. Phys. A: Math. Theor. 44 (2011), 295208, 26 pages, arXiv:1103.2943.

[9] Coste A., Gannon T., Ruelle P., Finite group modular data, Nuclear Phys. B 581 (2000), 679-717, hep-th/0001158.

[10] Covering groups of the alternating and symmetric groups, http://en.wikipedia.org/wiki/Covering_ groups_of_the_alternating_and_symmetric_groups.

[11] Curtis C.W., Reiner I., Representation theory of finite groups and associative algebras, Pure and Applied Mathematics, Vol. 11, Interscience Publishers, New York - London, 1962.

[12] Di Francesco P., Zuber J.-B., SU( $N)$ lattice integrable models associated with graphs, Nuclear Phys. B 338 (1990), 602-646.

[13] Dijkgraaf R., Pasquier V., Roche P., Quasi Hopf algebras, group cohomology and orbifold models, Nuclear Phys. B Proc. Suppl. 18 (1991), 60-72.

[14] Dijkgraaf R., Vafa C., Verlinde E., Verlinde H., The operator algebra of orbifold models, Comm. Math. Phys. 123 (1989), 485-526.

[15] Drinfeld V.G., Quasi-Hopf algebras, Leningrad Math. J. 1 (1989), 1419-1457.

[16] Drinfeld V.G., Quasi-Hopf algebras and Knizhnik-Zamolodchikov equations, Preprint ITP-89-43E, Kiev, 1989.

[17] Drinfeld V., Gelaki S., Nikshych D., Ostrik V., On braided fusion categories. I, Selecta Math. (N.S.) 16 (2010), 1-119, arXiv:0906.0620.

[18] Fairbairn W.M., Fulton T., Klink W.H., Finite and disconnected subgroups of $\mathrm{SU}_{3}$ and their application to the elementary-particle spectrum, J. Math. Phys. 5 (1964), 1038-1051.

[19] Feng B., Hanany A., He Y.-H., Prezas N., Discrete torsion, non-abelian orbifolds and the Schur multiplier, J. High Energy Phys. 2001 (2001), no. 1, 033, 25 pages, hep-th/0010023.

[20] Finch P.E., Dancer K.A., Isaac P.S., Links J., Solutions of the Yang-Baxter equation: descendants of the six-vertex model from the Drinfeld doubles of dihedral group algebras, Nuclear Phys. B 847 (2011), 387-412, arXiv:1003.0501.

[21] Flyvbjerg H., Character table for the 1080-element point-group-like subgroup of SU(3), J. Math. Phys. 26 (1985), 2985-2989.

[22] Gannon T., Modular data: the algebraic combinatorics of conformal field theory, math.QA/0103044.

[23] GAP - Groups, Algorithms, Programming - a system for computational discrete algebra, http://www. gap-system.org.

[24] Ginsparg P., Curiosities at $c=1$, Nuclear Phys. B 295 (1988), 153-170.

[25] Grimus W., Ludl P.O., Principal series of finite subgroups of SU(3), arXiv:1006.0098.

[26] Groupprops, The group properties Wiki, http://groupprops.subwiki.org.

[27] Hayashi T., A canonical Tannaka duality for finite semisimple tensor categories, math.QA/9904073.

[28] Hoffman P.N., Humphrey J.F., Projective representations of the symmetric groups. $Q$-functions and shifted tableaux, Oxford Mathematical Monographs, Oxford Science Publications, The Clarendon Press, Oxford University Press, New York, 1992.

[29] Hu X., Jing N., Cai W., Generalized McKay quivers of rank three, arXiv:1207.2823.

[30] Huang J.-S., Lectures on representation theory, World Scientific Publishing Co. Inc., River Edge, NJ, 1999.

[31] Kac V.G., Todorov I.T., Affine orbifolds and rational conformal field theory extensions of $W_{1+\infty}$, Comm. Math. Phys. 190 (1997), 57-111, hep-th/9612078.

[32] Koornwinder T.H., Schroers B.J., Slingerland J.K., Bais F.A., Fourier transform and the Verlinde formula for the quantum double of a finite group, J. Phys. A: Math. Gen. 32 (1999), 8539-8549, math.QA/9904029. 
[33] Ludl P.O., On the finite subgroups of U(3) of order smaller than 512, J. Phys. A: Math. Theor. 43 (2010), 395204, 28 pages, Corrigendum, J. Phys. A: Math. Theor. 44 (2011), 139501, arXiv:1006.1479.

[34] Ludl P.O., Systematic analysis of finite family symmetry groups and their application to the lepton sector, arXiv:0907.5587.

[35] Luhn C., Nasri S., Ramond P., Tri-bimaximal neutrino mixing and the family symmetry $\mathbb{Z}_{7} \rtimes \mathbb{Z}_{3}$, Phys. Lett. B 652 (2007), 27-33, arXiv:0706.2341.

[36] Lusztig G., Exotic Fourier transform, Duke Math. J. 73 (1994), 227-241.

[37] Lusztig G., Unipotent representations of a finite Chevalley group of type E8, Quart. J. Math. Oxford Ser. (2) 30 (1979), 315-338.

[38] Mathematica, Version 9.0, Wolfram Research, Inc., Champaign, IL, 2012, http://www.wolfram.com.

[39] McKay J., Graphs, singularities, and finite groups, in The Santa Cruz Conference on Finite Groups (Univ. California, Santa Cruz, Calif., 1979), Proc. Sympos. Pure Math., Vol. 37, Amer. Math. Soc., Providence, R.I., 1980, 183-186.

[40] Ocneanu A., Paths on Coxeter diagrams: from Platonic solids and singularities to minimal models and subfactors, Fields Institute Monographs, Amer. Math. Soc., Providence, RI, 1999.

[41] Ogievetsky O., Private communication, 1995.

[42] Ostrik V., Module categories over the Drinfeld double of a finite group, Int. Math. Res. Not. 2003 (2003), 1507-1520, math.QA/0202130.

[43] Ostrik V., Module categories, weak Hopf algebras and modular invariants, Transform. Groups 8 (2003), 177-206, math.QA/0111139.

[44] Parattu K.M., Wingerter A., Tribimaximal mixing from small groups, Phys. Rev. D 84 (2011), 013011, 17 pages, arXiv:1012.2842.

[45] Petkova V.B., Zuber J.-B., The many faces of Ocneanu cells, Nuclear Phys. B 603 (2001), 449-496, hep-th/0101151.

[46] Roan S.-S., Minimal resolutions of Gorenstein orbifolds in dimension three, Topology 35 (1996), 489-508.

[47] Schweigert C., Private communication.

[48] Thompson M., Towards topological quantum computation? - Knotting and fusing flux tubes, arXiv:1012.5432.

[49] Vafa C., Modular invariance and discrete torsion on orbifolds, Nuclear Phys. B 273 (1986), 592-606.

[50] Verlinde E., Fusion rules and modular transformations in 2D conformal field theory, Nuclear Phys. B 300 (1988), 360-376.

[51] Witherspoon S.J., The representation ring of the quantum double of a finite group, Ph.D. thesis, The University of Chicago, 1994, available at www.math.tamu.edu/ sjw/pub/SJWthesis.pdf.

[52] Yau S.S.-T., Yu Y., Gorenstein quotient singularities in dimension three, Mem. Amer. Math. Soc. 105 (1993), viii+88 pages.

[53] Yu J., A note on closed subgroups of compact Lie groups, arXiv:0912.4497. 